ARTICLE

DOI: $10.1038 / s 41467-018-04850-0$

\title{
ATR is a multifunctional regulator of male mouse meiosis
}

Alexander Widger ${ }^{1}$, Shantha K. Mahadevaiah', Julian Lange ${ }^{2}$, Elias Ellnati ${ }^{1}$, Jasmin Zohren', Takayuki Hirota (D) ${ }^{1}$, Sarai Pacheco (10 3,4, Andros Maldonado-Linares ${ }^{3,4}$, Marcello Stanzione ${ }^{5}$, Obah Ojarikre ${ }^{1}$, Valdone Maciulyte ${ }^{1}$, Dirk G. de Rooij ${ }^{6}$, Attila Tóth $^{5}$, Ignasi Roig (1D ${ }^{3,4}$, Scott Keeney (D) ${ }^{2}$ \& James M.A. Turner ${ }^{1}$

Meiotic cells undergo genetic exchange between homologs through programmed DNA double-strand break (DSB) formation, recombination and synapsis. In mice, the DNA damage-regulated phosphatidylinositol-3-kinase-like kinase (PIKK) ATM regulates all of these processes. However, the meiotic functions of the PIKK ATR have remained elusive, because germline-specific depletion of this kinase is challenging. Here we uncover roles for ATR in male mouse prophase I progression. ATR deletion causes chromosome axis fragmentation and germ cell elimination at mid pachynema. This elimination cannot be rescued by deletion of ATM and the third DNA damage-regulated PIKK, PRKDC, consistent with the existence of a PIKK-independent surveillance mechanism in the mammalian germline. ATR is required for synapsis, in a manner genetically dissociable from DSB formation. ATR also regulates loading of recombinases RAD51 and DMC1 to DSBs and recombination focus dynamics on synapsed and asynapsed chromosomes. Our studies reveal ATR as a critical regulator of mouse meiosis.

\footnotetext{
${ }^{1}$ Sex Chromosome Biology Lab, The Francis Crick Institute, 1 Midland Road, London NW1 1AT, UK. ${ }^{2}$ Molecular Biology Program, Howard Hughes Medical Institute, Memorial Sloan Kettering Cancer Center, New York, NY 10065, USA. ${ }^{3}$ Genome Integrity and Instability Group, Institut de Biotecnologia i Biomedicina, Universitat Autònoma de Barcelona, Cerdanyola del Vallès, Barcelona 08193, Spain. ${ }^{4}$ Department of Cell Biology, Physiology and Immunology, Cytology and Histology Unit, Universitat Autònoma de Barcelona, Cerdanyola del Vallès, Barcelona 08193, Spain. ${ }^{5}$ Institute of Physiological Chemistry, Faculty of Medicine at the TU Dresden, Fetscherstraße 74, 01307 Dresden, Germany. ${ }^{6}$ Center for Reproductive Medicine, Academic Medical Center, University of Amsterdam, Amsterdam 1105 AZ, The Netherlands. Correspondence and requests for materials should be addressed to J.M.A.T. (email: james.turner@crick.ac.uk)
} 
TR is a serine-threonine kinase with ubiquitous functions in somatic genome stability and checkpoint control ${ }^{1}$. Studies on non-mammalian organisms have revealed that ATR is also essential for meiosis. ATR orthologs regulate meiotic double-strand break (DSB) resection ${ }^{2}$, stoichiometry of DSB-associated strand-exchange proteins RAD51 and $\mathrm{DMCl}^{3}$, inter-homolog bias ${ }^{4,5}$ and crossover formation ${ }^{6}$. They are also components of prophase I checkpoints that ensure centromere pairing $^{7}$, timely repair of recombination intermediates ${ }^{8,9}$ and correct coupling of DNA replication with DSB induction ${ }^{10,11}$. In humans, hypomorphic Atr mutations cause Seckel syndrome, a pleiotropic, autosomal recessive disorder associated with

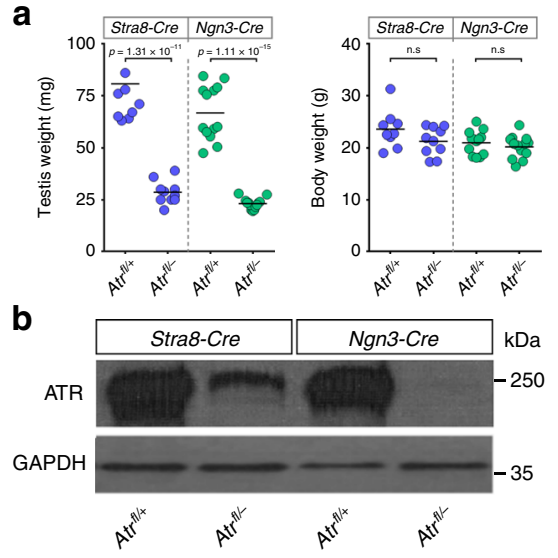

C
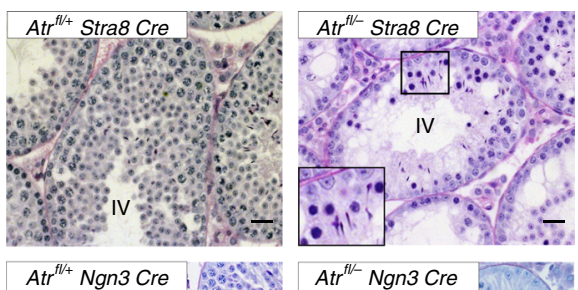

$\mathrm{Atr}^{\mathrm{fl} / \mathrm{N}} \mathrm{Ngn} 3 \mathrm{Cre}$
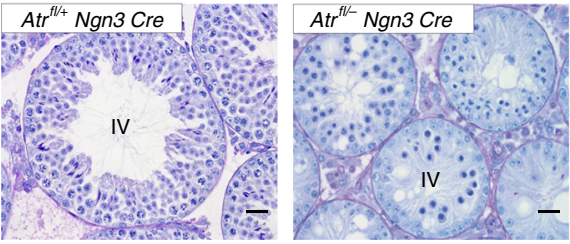

$\operatorname{Atr}^{f l /+}$
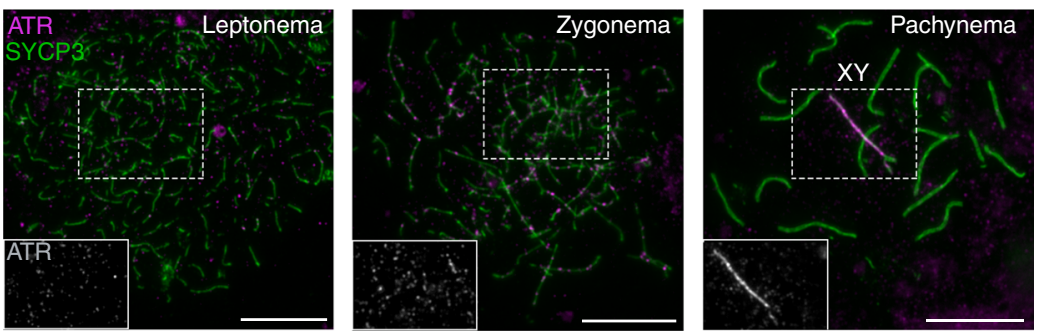

$\operatorname{Atr}^{f l /}$
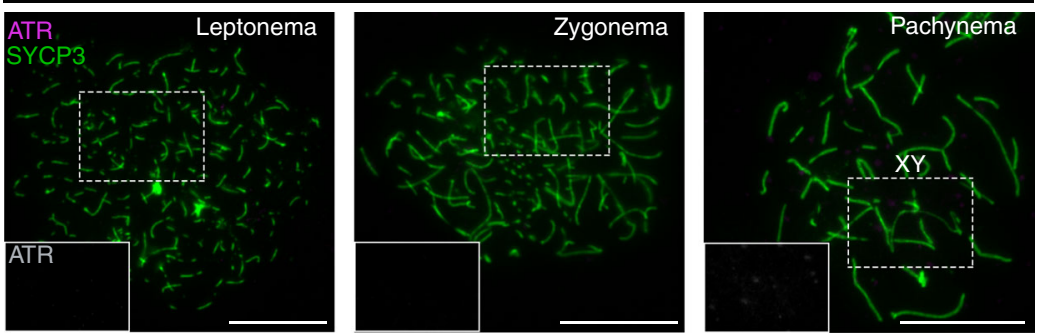

Atr $^{f l /+}$
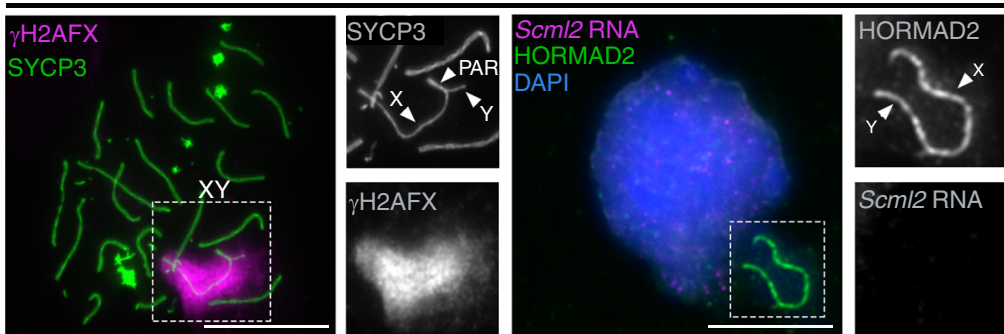

Scml2 RNA

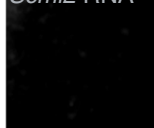

g

Atr $^{f l-}$
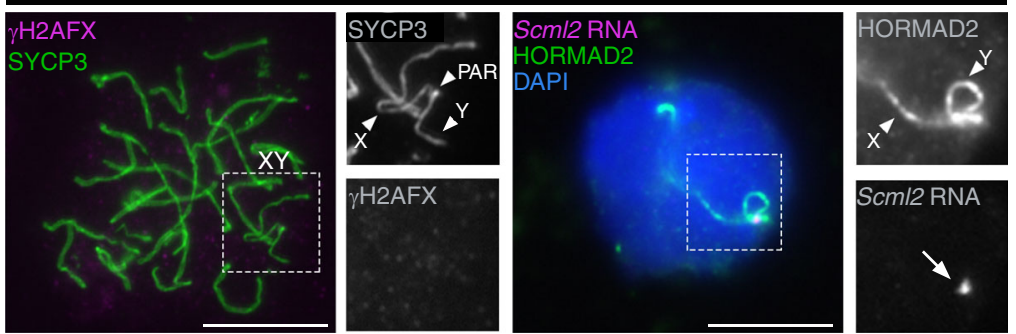
dwarfism, craniofacial abnormalities, intellectual disability and cryptorchidism ${ }^{12}$. In human cancer cell lines, ATR haploinsufficiency impairs the DNA damage response ${ }^{13}$. Determining the functions of ATR in mouse meiosis has been challenging. Heterozygous Atr deletion compromises postnatal survival ${ }^{14}$ and homozygous deletion causes embryonic lethality ${ }^{14}, 15$. An inducible Cre-ERT2 approach recently revealed that ATR regulates meiotic sex chromosome inactivation (MSCI), the silencing of the $\mathrm{X}$ and $\mathrm{Y}$ chromosomes in male meiosis, via serine-139 $\mathrm{H} 2 \mathrm{AX}$ phosphorylation $(\gamma \mathrm{H} 2 \mathrm{AX})^{16}$. However, this method resulted in partial rather than complete ATR depletion.

Here we describe a superior conditional strategy for dissecting additional meiotic ATR functions. Using this approach, we show that ATR regulates homologous synapsis as well as multiple steps in recombination. By generating mutants deficient in both ATR and ATM, we identify shared and distinct functions for these kinases in mouse meiosis.

\section{Results}

A strategy for efficient meiotic Atr depletion. For this purpose, we generated male mice carrying one Atr floxed $(A t r f l)$ allele, in which the exon 44 kinase domain of Atr is flanked by loxP sites ${ }^{17}$, and one Atr-null $\left(A t r^{-}\right)$allele, in which the first three coding exons of Atr are replaced by a neomycin selection cassette ${ }^{14}$. The resulting $A t_{r} \mathrm{fll}^{\mathrm{l}-}$ males also carried a transgene expressing Cre recombinase under the control of either a Stra 8 or Ngn3 promoter fragment. Stra8-Cre is expressed from $\mathrm{P} 3$ (postnatal day 3$)^{18}$, while Ngn3-Cre is expressed from P7 $7^{19}, 20$. Testis weights at P30 were reduced three- to fourfold in Atr fl/- Stras-Cre males and Atr $f^{f l-}$ Ngn3-Cre males relative to Atr $\mathrm{fl}^{\mathrm{fl}+}$ Cre-carrying (i.e., Atr heterozygous) controls, while body weights were unaffected (Fig. 1a). We observed no difference in testis weights between Atr $\mathrm{fl} /+$ males carrying Cre transgenes and those not carrying Cre transgenes (Fig. 1 legend). Western blotting showed that ATR protein was reduced in Atr ${ }^{f l} /$ - Stra8-Cre testes, and even more so in Atrfll- Ngn3-Cre testes (Fig. 1b). This finding supports previous evidence that the majority of testis ATR expression occurs in spermatocytes ${ }^{16,21}$. Testis histology revealed germ cell failure at seminiferous tubule stage IV, corresponding to mid pachynema of meiosis, in both Cre models (Fig. 1c), reminiscent of findings in $A t^{f l l-}$ Cre-ERT2 mice ${ }^{16}$. However, the stage IV elimination was clearly less robust in Atr fl/-Stra8-Cre than Atr ${ }^{f l /-}$ Ngn3-Cre males, because elongating spermatids were observed in some testis sections from the former but not latter genotype (Fig. 1c inset). We therefore focused on Atr fl/- Ngn3-Cre mice (hereafter Atroll-), with Atr fl/ Ngn3-Cre (hereafter Atr ${ }^{f l /+}$ ) serving as controls.

Combined immunofluorescence for ATR and the axial element protein SYCP ${ }^{22}$ confirmed that the characteristic ATR staining pattern observed in control leptotene, zygotene and pachytene spermatocytes (Fig. 1d) was absent in Atr ${ }^{f l /-}$ males (Fig. 1e). Furthermore, MSCI, assayed at early pachynema by acquisition of $\gamma \mathrm{H} 2 \mathrm{AX}$ on the XY bivalent and RNA fluorescent in situ hybridization (FISH) to detect absence of expression of the X-chromosome gene $\mathrm{Scml2}$, was present in control males (Fig. 1f) but abolished in Atrfl/- males (Fig. 1g). Thus, by multiple criteria, $A t r^{f l /-}$ males exhibited efficient ATR depletion.

At stage IV, when wild-type spermatocytes reach mid pachynema, Atr fll- spermatocytes contained highly fragmented chromosome axes and nucleus-wide $\gamma \mathrm{H} 2 \mathrm{AX}$ staining (Supplementary Fig. 1a; see Methods for meiotic staging criteria used throughout this study). These mid-pachytene Atr fll- cells were readily distinguishable from $A t^{f f l-}$ cells at leptonema, in which axial elements were shorter and uniform in length, and $\gamma \mathrm{H} 2 \mathrm{AX}$ staining across the nucleus was more heterogeneous (Supplementary Fig. 1b). Mid-pachytene axis fragmentation and nucleuswide $\gamma \mathrm{H} 2 \mathrm{AX}$ staining were also noted in $\mathrm{Atm}^{-1-}$ males (Supplementary Fig. 1c), as described previously ${ }^{23}$. Neither phenotype was observed in Spo11 ${ }^{-/-}, \mathrm{Dmcl}^{-/-}$and $\mathrm{Msh}^{-/-}$ males (Supplementary Fig. 1d-f), which display stage IV arrest. Instead, $\gamma \mathrm{H} 2 \mathrm{AX}$ in Spo11 ${ }^{-1-}$ spermatocytes was restricted to the transcriptionally inactive pseudosex body (Supplementary Fig. 1d), while in $D m c^{-1-}$ and $M s h 5^{--}$spermatocytes it formed axis-associated clouds (Supplementary Fig. 1e,f), consistent with published reports ${ }^{23-25}$. These findings suggested that midpachytene axis degeneration and nucleus-wide $\gamma \mathrm{H} 2 \mathrm{AX}$ staining are features of ATR and ATM (ataxia telangiectasia mutated) deletion, and not merely a consequence of stage IV germ cell death.

Mid-pachytene elimination in males deficient in Atr, Atm and Prkdc. We investigated mechanisms driving mid-pachytene elimination of $A t^{\mathrm{fl} / \mathrm{-}}$ spermatocytes. In male mice stage IV arrest in response to recombination defects is promoted by $\mathrm{ATM}^{26}$, whereas MSCI failure can cause stage IV elimination independently of $\mathrm{ATM}^{16,27}$. Since Atrfl/- males exhibit defective MSCI, we predicted that mid-pachytene germ cell loss would be preserved in mice doubly deficient for ATR and ATM, irrespective of whether ATR is involved in recombination.

To test this prediction, we examined testis histology in Atr $\mathrm{fl} /$ $\mathrm{Atm}^{-1-}$ mutants. As expected, double-mutant testes exhibited greatly reduced ATR and ATM protein levels (Fig. 2a, b). At leptonema, axis morphology was grossly unaffected (Fig. 2c, left panel; Supplementary Fig. 1g), but most spermatocytes at later stages exhibited axial fragmentation (Fig. 2c, right panel inset; Supplementary Fig. 1g). Thus, axis morphology was more severely compromised in double mutants than in either single mutant. Leptotene and zygotene H2AX phosphorylation are catalyzed by ATM and ATR, respectively $23,24,28,29$. In leptotene

Fig. 1 A conditional strategy for efficient depletion of ATR during male mouse meiosis. P30 testis and body weights (a), testis western blots (b), and periodic acid-Schiff and hemotoxylin/eosin-stained stage IV testis sections (c) in Atr fl/ + Stra8-Cre males $\left(n=9\right.$ males), Atr ${ }^{f l} /$ - Stra8-Cre males $(n=10$ males), Atr ${ }^{f l} /+$ Ngn3-Cre males ( $n=13$ males) and Atr ${ }^{f l}-$ Ngn3-Cre males ( $n=13$ males; means and $p$ values for a indicated; unpaired $t$-test). Testis weights

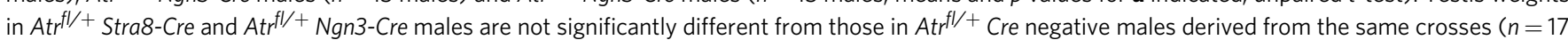
Atr ${ }^{\mathrm{I} /} \mathrm{I}+$ Cre negative males from the Stra8-Cre cross, $p=0.37, n=11$ Atr $\mathrm{fl}^{\mathrm{I}}+$ Cre negative males from the Ngn3-Cre cross, $\left.p=0.06\right)$. Inset in c shows presence of elongating spermatids in some tubules from Atr $\mathrm{fl}^{\mathrm{l}-\mathrm{S}} \mathrm{Stra8}$-Cre males. d, e ATR (magenta) and SYCP3 (green) staining in Atr $\mathrm{fl}^{\mathrm{I} /+} \mathrm{Ngn3} 3-\mathrm{Cre}$ (denoted Atr ${ }^{f l /+}$ ) and Atr ${ }^{f l /-}$ Ngn3-Cre (denoted Atr ${ }^{f l /}$ ) males. In Atrl//+ males ( $n=2$ males) ATR is observed as foci (see insets) in $85 \%$ of leptotene cells ( $n=20$ cells) and $82 \%$ of zygotene cells $(n=28$ cells), and on the asynapsed region of the XY bivalent in $100 \%$ of pachytene cells ( $n=30$ cells). In Atr $f / /-$ males ( $n=2$ males) ATR is observed in no cells at these three stages $(n=21,30$ and 32 cells at leptonema, zygonema and pachynema, respectively). $\mathbf{f}$ Validation of ATR depletion in Atr ${ }^{f l /-}$ by analysis of $\mathrm{MSCl}$. In Atr ${ }^{f l /+}$ males, all pachytene cells show coating of the $\mathrm{X}$ and $\mathrm{Y}$ chromosome (arrowheads) but not the pseuodautosomal regions (PAR, arrowhead) by $\gamma \mathrm{H} 2 \mathrm{AX}$ (magenta; left panels; $X Y$ bivalent in box magnified in smaller panels). This coating causes silencing of the X-chromosome gene Scm/2 (magenta; right panels) and compartmentalization of the XY bivalent (labeled with HORMAD2; green) in the sex body ( $n=$ one male, 29 cells; magnified in smaller panels). $\mathbf{g}$ In Atr fl/- males, XY chromosome $\gamma \mathrm{H} 2 \mathrm{AX}$ coating and sex body compartmentalization do not occur. As a result, Scml2 expression (arrow) persists in all early pachytene cells ( $n=$ one male; 30 cells). Scale bar in (c) $20 \mu \mathrm{m}$, other scale bars $10 \mu \mathrm{m}$ 
spermatocytes from Atr ${ }^{f l-}$ Atm ${ }^{-/-}$mutants, $\gamma \mathrm{H} 2 \mathrm{AX}$ was absent (Fig. 2c, left panel). Furthermore, zygotene spermatocytes that lacked axial fragmentation, and could thus be unambiguously staged, lacked $\gamma \mathrm{H} 2 \mathrm{AX}$ (Fig. 2c, middle panel). Thus, both phosphatidylinositol-3-kinase-like kinases (PIKKs) had been efficiently depleted in these double mutants. Consistent with our prediction, stage IV elimination was preserved in Atr fl/$\mathrm{Atm}^{-/-}$testes (Fig. 2d). In these males we observed a population of spermatocytes with nucleus-wide $\gamma \mathrm{H} 2 \mathrm{AX}$ staining. Based on their advanced axial fragmentation pattern (Fig. 2c, right panel) and adlumenal location within seminiferous tubule sections (Supplementary Fig. 1h), these spermatocytes were inferred to be at mid pachynema.

Our findings were consistent with a checkpoint-independent mechanism, most likely defective MSCI, driving germ cell loss in Atr fl $^{-} \mathrm{Atm}^{-1-}$ males. However, the remaining DNA damageregulated PIKK, PRKDC, was still present in these mutants and could contribute a checkpoint function. We therefore examined germ cell progression in males deficient in all three DNA damage-regulated PIKKs. For this experiment we used the Prkdc scid mutation ${ }^{30}$ and an Atm flox allele ${ }^{31}$, because combined homozygosity for Prkdc scid and the Atm-null mutation causes embryonic lethality ${ }^{32}$. In $A t r{ }^{f l-} A t m{ }^{f l /-} P r k d c^{s c i d / s c i d}$ males, testis ATM and ATR levels were depleted (Fig. 2a, b), and consequently ATM- and ATR-dependent $\gamma \mathrm{H} 2 \mathrm{AX}$ staining was absent (Fig. 2e, left and middle panels). Interestingly, pachytene nucleus-wide $\gamma \mathrm{H} 2 \mathrm{AX}$ staining was also abolished (Fig. 2e, right panel). Thus,
PRKDC mediates pachytene serine-139 H2AX phosphorylation in $A t r^{f l-} A_{t m}{ }^{f l-}$ males. Nevertheless, in the triple-mutant stage IV germ cell loss still occurred, and axis fragmentation was even more severe than in $A t r^{f l /-} A t m^{f l /-}$ males (Fig. 2e, right panel; Fig. 2f). Thus, mid-pachytene elimination persists in mice deficient in all three DNA damage-regulated PIKKs.

Atr regulates homologous synapsis. We also investigated synapsis and recombination in Atrt/- males, and where possible we compared findings in this mutant to those in $\mathrm{Atr}^{\mathrm{fl} /-} \mathrm{Atm} \mathrm{fl}^{\mathrm{fl}}$ males. To address whether ATR regulates homologous synapsis, we immunostained for SYCP3 and the asynapsis marker HORMAD2. We focused on $A t r f l-$ cells at early pachynema, i.e., prior to extensive axial element fragmentation (Supplementary Fig. 1a). Synapsis was normal in $86 \%(n=104)$ of early pachytene cells from control males, a frequency similar to that in $A t r f l+$ males without the Ngn3-Cre transgene $(89 \% ; n=100)$. As expected, in control cells HORMAD2 was absent on the autosomal bivalents and present on the non-homologous, asynapsed regions of the $\mathrm{X}$ and $\mathrm{Y}$ chromosome in these instances (Fig. 3a). Furthermore, in these cells, non-homologous synapsis between the sex chromosomes and the autosomes was not observed. However, in Atr flmales, only $20 \%(n=107)$ of early pachytene cells achieved complete homologous synapsis. The remaining cells exhibited varying degrees of asynapsis affecting the XY pair and the autosomes (Fig. 3b; see below for details). In addition, $\mathrm{X}$ and $\mathrm{Y}$
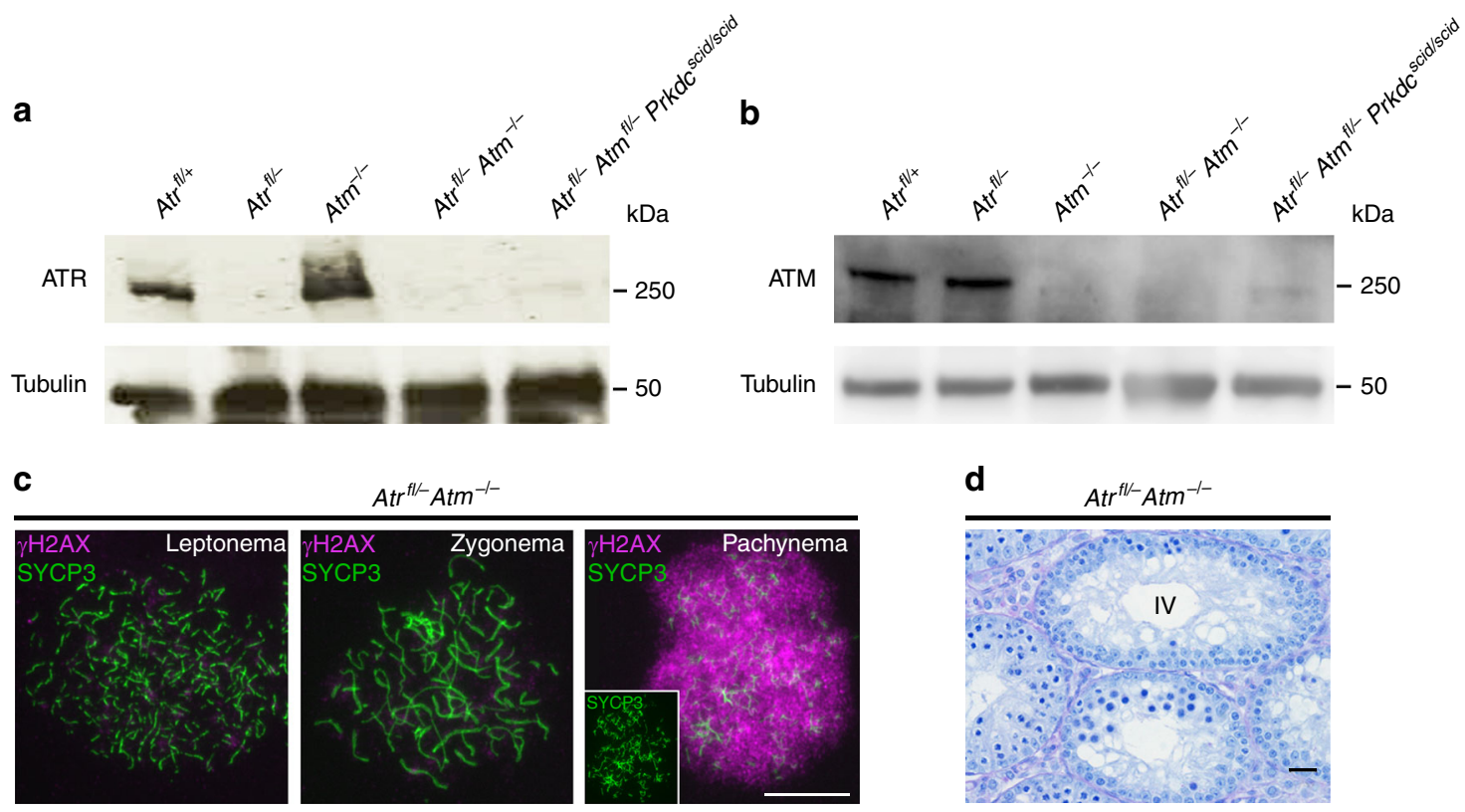

$\operatorname{Atr}^{\mathrm{fl} /-} \mathrm{Atm}^{-1-}$

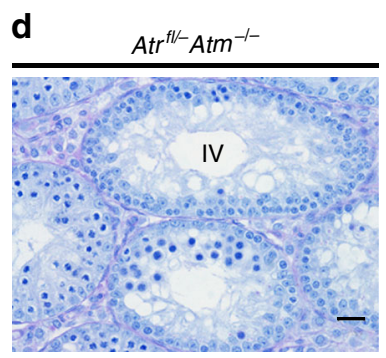

e
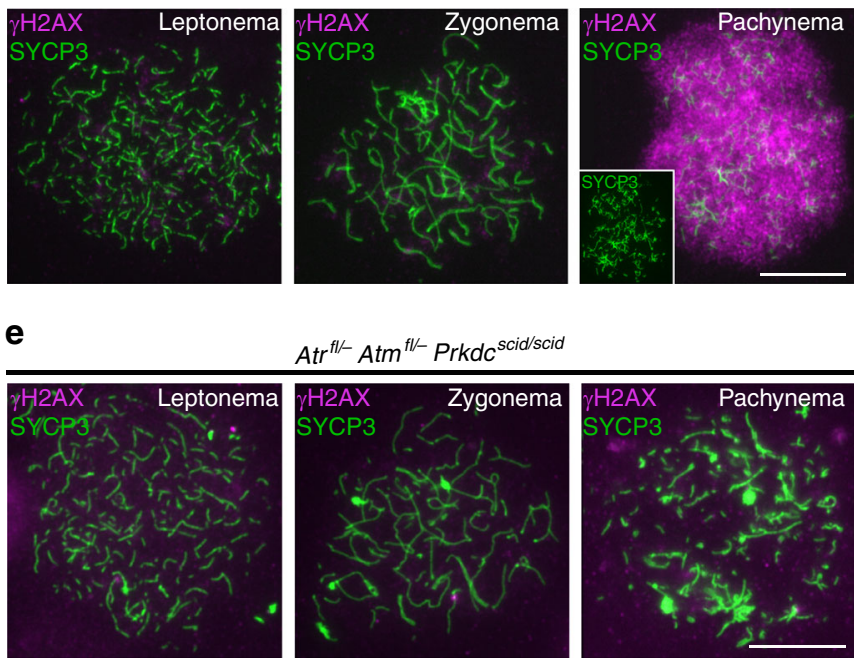

$\operatorname{Atr}^{f l-} \operatorname{Atm}^{f l-}$ Prkdc scid/scid
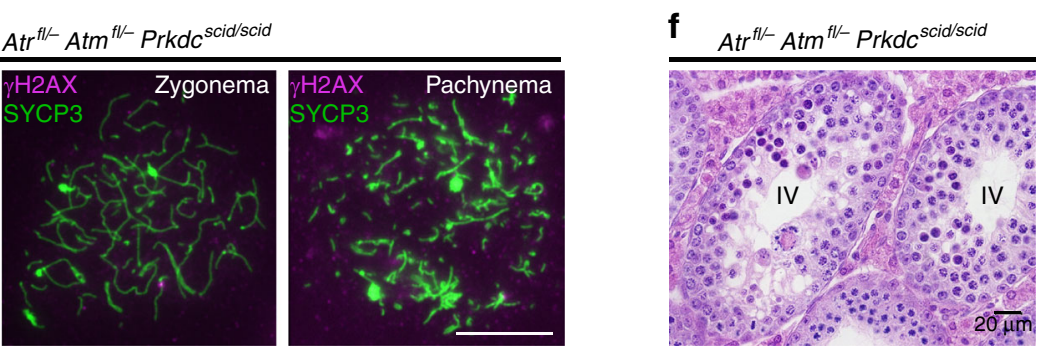

Fig. 2 Mid-pachytene germ cell elimination is preserved in mice deficient in the PIKKs. Western blot showing a ATR and $\mathbf{b}$ ATM depletion in mice with different PIKK mutations. c $\gamma \mathrm{H} 2 \mathrm{AX}$ (magenta) and SYCP3 (green) immunostaining ( $n=2$ males, 25 cells for each stage) and $\mathbf{d}$ stage IV elimination in Atr $\mathrm{fl}^{\mathrm{f}}$ $\mathrm{Atm}^{-/-}$males. e $\gamma \mathrm{H} 2 \mathrm{AX}$ and SYCP3 immunostaining ( $n=2$ males, 25 cells for each stage) and $\mathbf{f}$ stage IV elimination in $\mathrm{Atr} \mathrm{fl}^{\mathrm{fl}-} \mathrm{Atm} \mathrm{fl} /-^{-} \mathrm{Prkd} \mathrm{Csid}^{\mathrm{scid}}$ males. Scale bar $20 \mu \mathrm{m}$ in $\mathbf{d}, \mathbf{f}$ and $10 \mu \mathrm{m}$ in $\mathbf{c}$, e 
self-synapsis and non-homologous synapsis between the sex chromosomes and autosomes were more common in $\mathrm{Atr} f \mathrm{fl}-$ cells (Fig. 3b). In mice, the XY pseudoautosomal regions (PARs) undergo late synapsis and DSB formation ${ }^{33}$, and in yeast the homolog bias of late-forming DSBs is partially dependent on
$\mathrm{ATR}^{2}$. We therefore determined whether asynapsis in $A t r f l-$ males more often affects the sex chromosomes than the autosomes. We identified the sex chromosomes using DNA FISH (Slx probe for X and Sly probe for Y; Fig. 3a, b; insets). In Atr fl/- males, $77 \%$ of early pachytene cells exhibited XY asynapsis, while $59 \%$

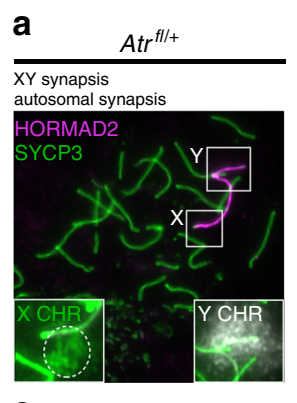

b

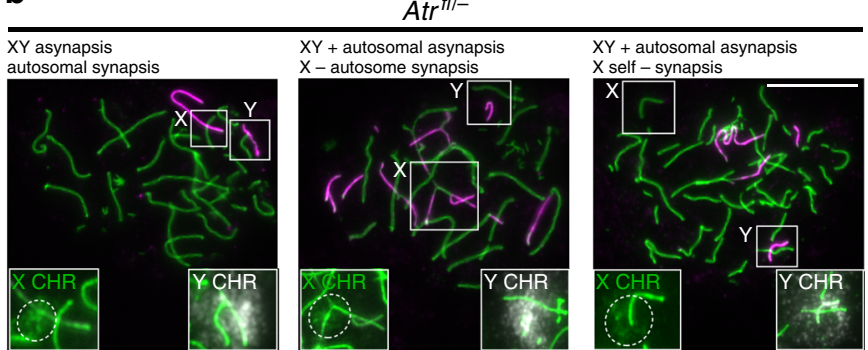

C

Spo11 $11^{-1-}$

d
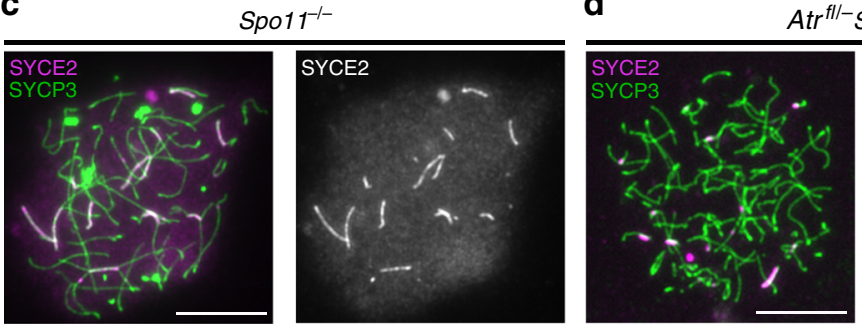

Po11 $11^{-1-}$

e
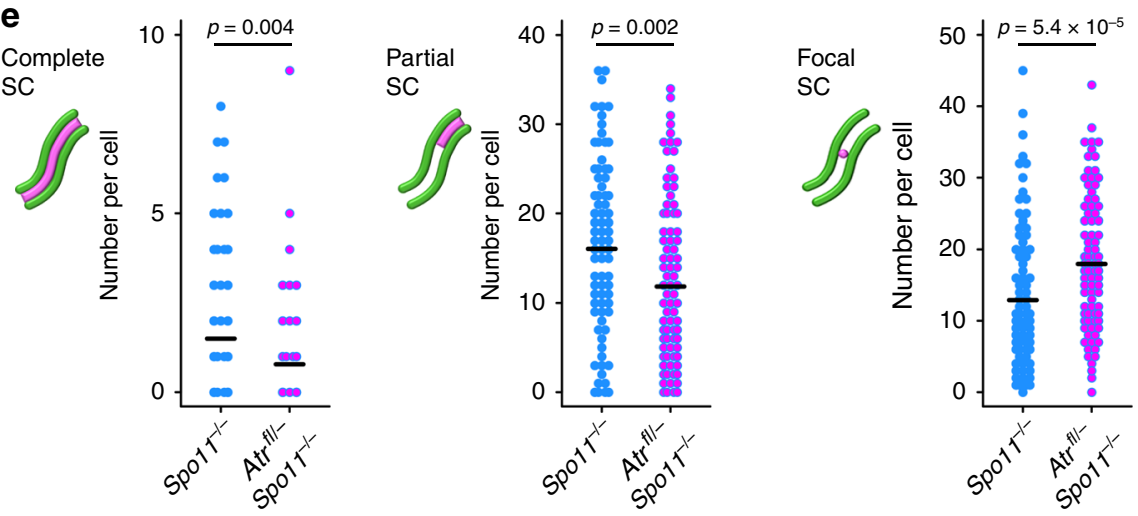

$\mathbf{f}$

$\mathbf{g}$
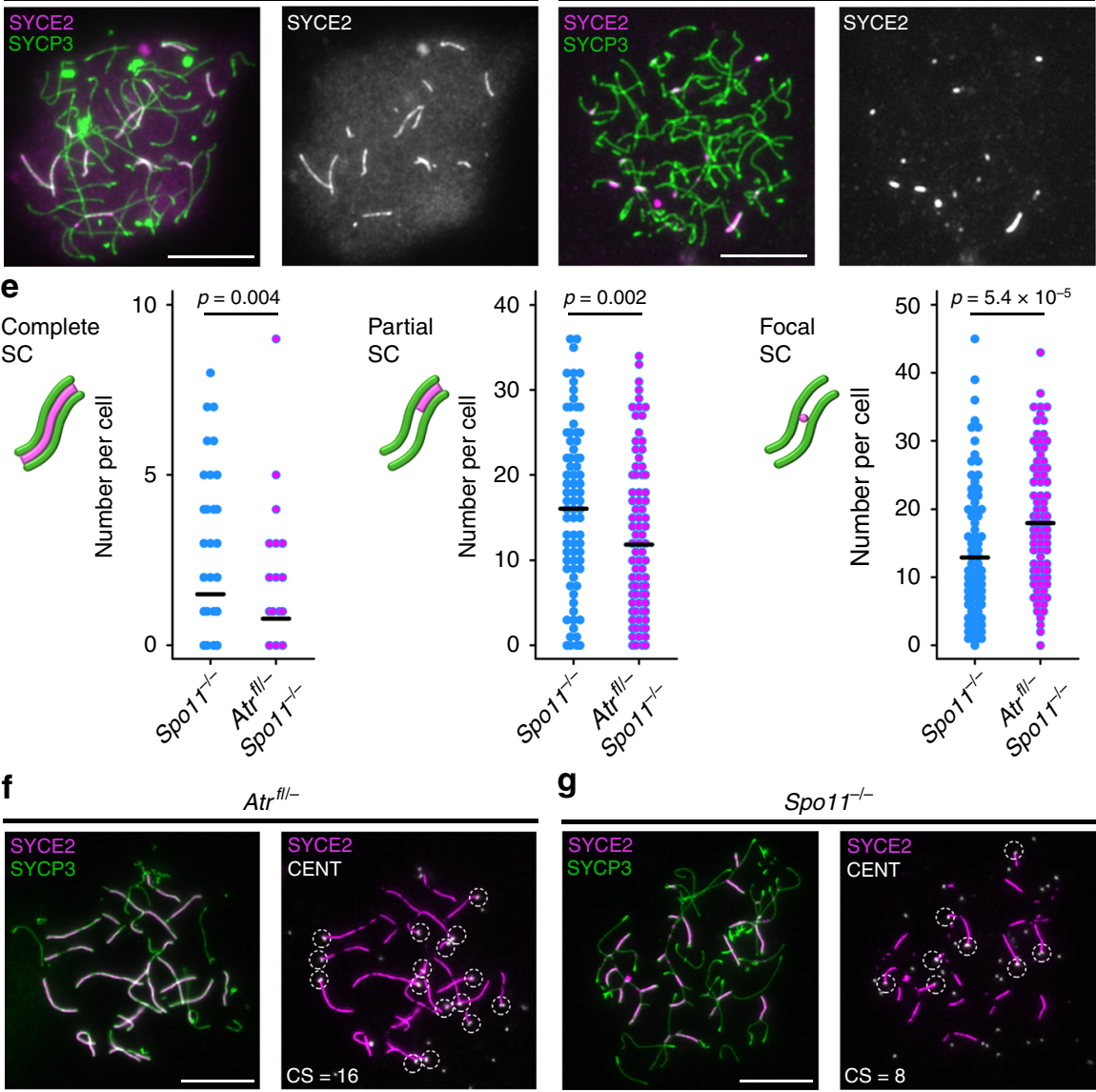

Spo1 $11^{-1-}$
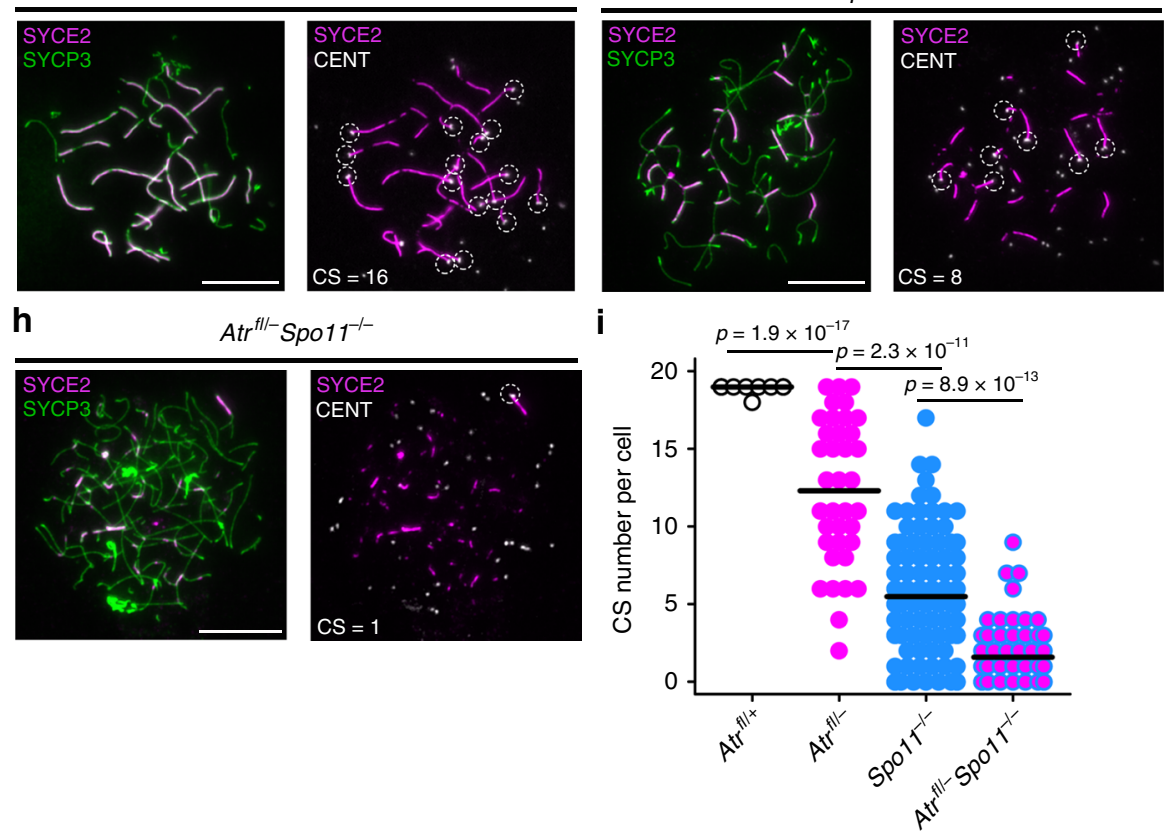
exhibited autosomal asynapsis (see legend for further details). In control males, $13 \%$ of early pachytene cells exhibited XY asynapsis and $13 \%$ autosomal asynapsis. Thus, ATR deletion has a more deleterious effect on XY than on autosomal synapsis.

Asynapsis can result from defects in synaptonemal complex (SC) formation or recombination. To address whether ATR can promote SC formation independent of recombination, we used the Spo11-null mutation, which permits genetic dissociation of synapsis from recombination initiation ${ }^{34}$. Spo $11^{-/-}$males do not form programmed DSBs, yet achieve extensive SC formation between non-homologs 35,36 . If deletion of ATR impeded SC formation in Spo11 nulls, then ATR must have a role in SC formation. We therefore compared synapsis between Spo11 ${ }^{-/-}$ and Atr $f l-$ Spo11 $11^{-/}$males. Like each single mutant, Atr $f l-$ Spo11 ${ }^{-/-}$males exhibited stage IV germ cell elimination (Supplementary Fig. 2a,b). We classified SC formation, assessed using SYCP3 and the SC central element component SYCE2 ${ }^{37}$, into three classes: (i) complete SC, encompassing the entire axis length, (ii) partial SC, extending along only part of an axis, and (iii) focal SC (Fig. 3c-e). Relative to Spo11 ${ }^{-1-}$ males (Fig. 3c), Atr ${ }^{f l-}$ Spo11 $1^{-/}$males (Fig. 3d) exhibited a decrease in complete and partial SC formation, and an increase in focal SC (Fig. 3c-e). These findings suggest that ATR promotes conversion of SC foci into longer SC stretches. Using Atr fl/- Spo $11^{-/-}$males, we also demonstrated that formation of the pseudosex body in Spo11 ${ }^{-/-}$ males is ATR dependent (Supplementary Fig. 2c, d).

Our data suggested that ATR can promote synapsis independent of recombination. To strengthen these findings, we devised an additional method to quantify synapsis. We triple immunostained cells for SYCP3, SYCE2 and centromeres, and counted the number of centromeres that had achieved synapsis, i.e., that colocalized with SYCE2 signals (centromere-SYCE2, or CS number; Fig. 3f-i). The mean CS number was reduced in $A t r f l-$ relative to control males, confirming that ATR is required for normal levels of synapsis (Fig. 3f, i). In Atr fl/- Spo $11^{-/-}$males, the mean CS number was reduced relative to that in $S p o 11^{-/-}$males (Fig. 3g-i). Thus, ATR promotes SC formation both in the presence and absence of SPO11-generated DSBs. We did not compare synapsis in $A t r^{f l /-}$ males with that in $\mathrm{Atr}^{\mathrm{fl} /-} \mathrm{Atm}^{-/-}$ males, because in the latter model chromosome axes were highly fragmented at early pachynema.

Atr deletion does not influence DSB abundance. We further examined roles of ATR in DSB formation. Orthologs of ATM influence DSB homeostasis by acting as negative regulators of DSB induction ${ }^{38-42}$. In Saccharomyces cerevisiae ATR promotes DSB formation indirectly by increasing the length of prophase $\mathrm{I}^{43}$, but its impact on DSB levels in mammals is unknown. To address this question, we measured abundance of covalent
SPO11-oligonucleotide (SPO11-oligo) complexes, by-products of DSB induction, in testis extracts ${ }^{44}$. Consistent with previous work $^{40}$, in $\mathrm{Atm}^{-1-}$ testes SPO11-oligo complex levels were elevated relative to those observed in wild-type mice (Fig. $4 \mathrm{a}, \mathrm{b}$ ). However, SPO11-oligo complex levels in Atr fl/- testes, as well as in Atr fl/ males without the Ngn3-Cre transgene, were not detectably changed relative to controls (Fig. 4a, b). SPO11-oligo complex levels in $A t r^{f l-} \mathrm{Atm}^{-/}$testes were similar to those in $\mathrm{Atm}^{-/-}$testes $(p=0.29$; Fig. $4 \mathrm{a}, \mathrm{b})$. Thus, ATR and ATM have distinct functions with respect to DSB formation.

In addition to its role in DSB homeostasis, ATM is implicated in nucleolytic processing of $\mathrm{DSBs}^{40,45-47}$. SPO11 oligos are longer in $\mathrm{Atm}^{-1-}$ mice, with an increase in intermediate $(\sim 40-70$ nucleotide (nt)) and large (>300 nt) species at the expense of the small $(\sim 15-27 \mathrm{nt})$ ones observed in wild type ${ }^{40}$. SPO11-oligo size distribution in $A t r^{f l-}$ males and in $A t r^{f l+}$ males without the Ngn3-Cre transgene were not detectably changed relative to controls (Fig. 4c, d). However, in $A t r f l--\mathrm{Atm}^{-/}-$testes, SPO11 oligos were on average even larger than in $\mathrm{Atm}^{-/-}$testes, with a decrease in the abundance of small and intermediate oligos and a substantial increase in the amount of high-molecular-weight species running near the top of the gel (Fig. 4c, d). We conclude that ATR is largely dispensable for initial nucleolytic processing of SPO11-generated DSBs, but that it can partially compensate when ATM is not present to promote these events.

Atr controls RAD51 and DMC1 abundance at DSBs in leptonema. During leptonema, resected DSBs are coated with RPA (replication protein A) and the recombinases RAD51 and DMC1, which carry out strand invasion and recombinational repair. We used immunostaining to determine whether these early recombination components are influenced by ATR. Leptotene focus counts for RPA subunit 2 (hereafter termed RPA) were similar between $A t r f^{f l-}$ and controls (Fig. 4e, f). This finding supports conclusions from SPO11-oligo complex quantification that ATR does not influence DSB abundance (Fig. 4a, b), and is consistent with RPA acting upstream of $\mathrm{ATR}^{48}$. In addition, RPA counts in controls did not differ from those in Atr $f /+$ males without the Ngn3-Cre transgene (Fig. 4 legend). Importantly though, in Atr fl- males, RAD51 counts were reduced by almost half relative to controls (Fig. $4 \mathrm{~g}, \mathrm{~h}$ ). DMC1 counts were lower by a similar magnitude (Fig. 4i, j). Thus, in Atr ${ }^{f l-}$ males, DSB abundance appears grossly unaffected, but recombinase localization is compromised.

We also quantified RPA2, RAD51 and DMC1 foci at leptonema in $\mathrm{Atm}^{-1-}$ and $\mathrm{Atr} \mathrm{fl}^{-} \mathrm{Atm}^{-/-}$males. In both models, RPA counts were elevated twofold relative to $A t r f l--$ and control males (Fig. 4e, f). In Atm ${ }^{-/-}$males, RAD51 and DMC1 focus counts were similar to controls (Fig. $4 \mathrm{~g}-\mathrm{j}$ ). The failure of

\footnotetext{
Fig. 3 ATR is required for homologous synapsis. Examples of early pachytene synaptic outcomes in a Atr ${ }^{f l /+}$ males $(n=3$ males $)$ and $\mathbf{b}$ Atr ${ }^{f l /-}$ males $(n=3$ males) assessed using HORMAD2 (magenta), SYCP3 (green) and subsequent DNA FISH using Slx and Sly probes (labeled in insets as X chromosome in green and $Y$ chromosome in white). The Slx probe hybridizes to a sub-region of the $X$ chromosome (circled), while the Sly probe coats the majority of the $Y$ chromosome. In Atr $\mathrm{fl}^{\mathrm{l} /+}$ males (a), both the autosomes and the XY PARs are synapsed, while the non-homologous regions of the XY pair are asynapsed. In $\operatorname{Atr}^{\mathrm{fl} / \mathrm{+}}$ males ( $n=104$ cells, 2 males), 90 cells had normal synapsis, 12 cells had asynapsis of both the XY and autosomes, 1 cell had asynapsis only of the $\mathrm{XY}$ and 1 cell had asynapsis only of the autosomes. $\mathbf{b}$ Three examples of synaptic defects in Atr ${ }^{\mathrm{fl} / \text { - }}$ males, each described above respective image. In Atr $\mathrm{fl}^{\mathrm{I}-\mathrm{-}}$ males ( $n=107$ cells, 2 males), 21 cells had normal synapsis, 59 cells had asynapsis of both the $X Y$ and autosomes, 23 cells had asynapsis only of the $X Y, 4$ cells had asynapsis only of the autosomes, 10 cells had $X$ self-synapsis, 11 cells had $Y$ self-synapsis and 7 had non-homologous synapsis between the $X$

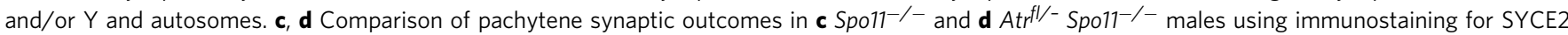
(magenta) and SYCP3 (green). e Quantitation of complete, partial and focal SC in Spo11 ${ }^{-/}$males ( $n=2$ males; 88 cells) and Atr ${ }^{f l /-}$ Spo $11^{-/-}$males ( $n=2$ males; 96 cells). Means and $p$ values (unpaired $t$-test) indicated. f-h Epistasis analysis of SPO11 and ATR in synapsis using the same markers plus immunostaining for centromeres (CENT; white) to determine CS number. For each cell, colocalizing SYCE2-CENT signals are indicated with dashed circles, and resulting CS numbers are shown. i CS number in Atr ${ }^{f l /}+$ males ( $n=2$ males; 55 cells), Atr ${ }^{f l /-}$ males $\left(n=2\right.$ males; 37 cells), Spo11 ${ }^{-1-}$ males $(n=2$ males; 114 cells) and $A$ trl $^{\mathrm{I} /-}$ Spo11 ${ }^{-/-}$males $(n=2$ males; 64 cells). Mean values and $p$ values (Mann-Whitney test) indicated. Scale bars $10 \mu \mathrm{m}$
} 
recombination focus counts to fully account for the large increase in DSB formation in the absence of ATM has been proposed to reflect inability of cytological methods to resolve the clustered DSBs that form nearby on the same chromatid or pair of sister chromatids ${ }^{40,42}$. However, in $\mathrm{Atr}^{\mathrm{fl}-\mathrm{Atm}} \mathrm{At}^{-/}$males RAD51 and
DMC1 counts were reduced to levels even lower than that observed in Atr fl/- males (Fig. $4 \mathrm{~g}-\mathrm{j}$ ). Thus, RPA counts are increased by deleting ATM but not ATR, while RAD51 and DMC1 counts are decreased by deleting ATR, and even more so by deleting both ATR and ATM.

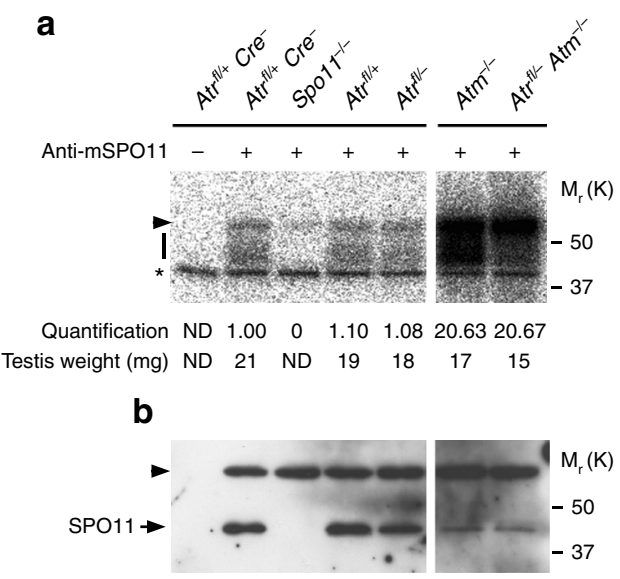

e
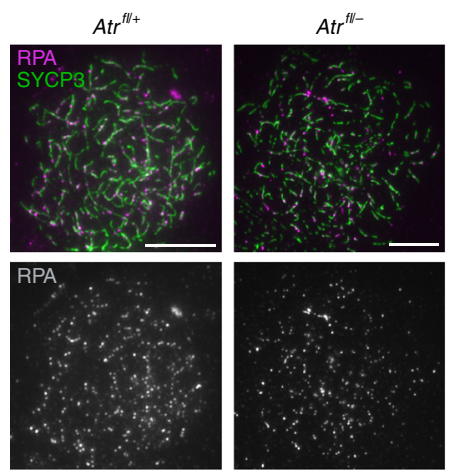

$\mathbf{g}$
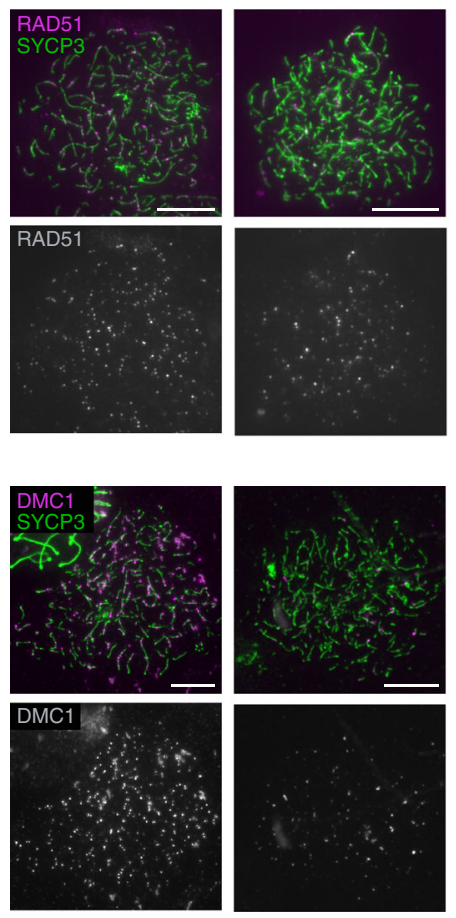

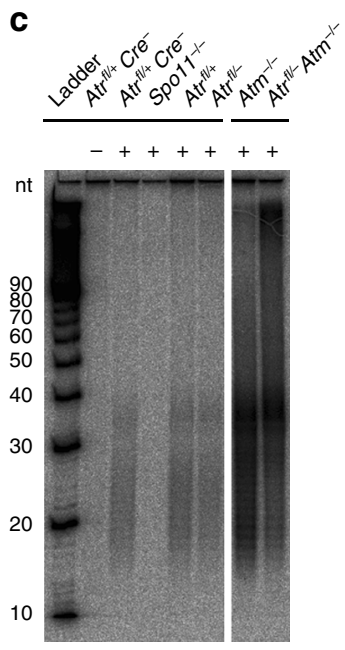

d

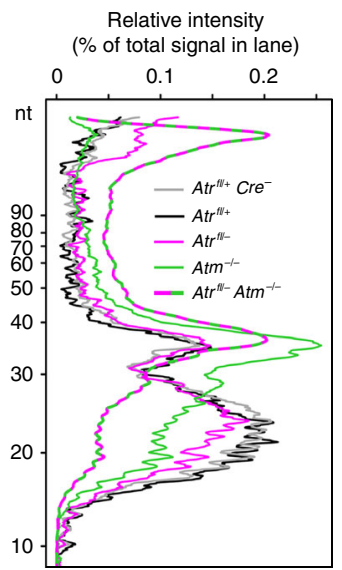

f
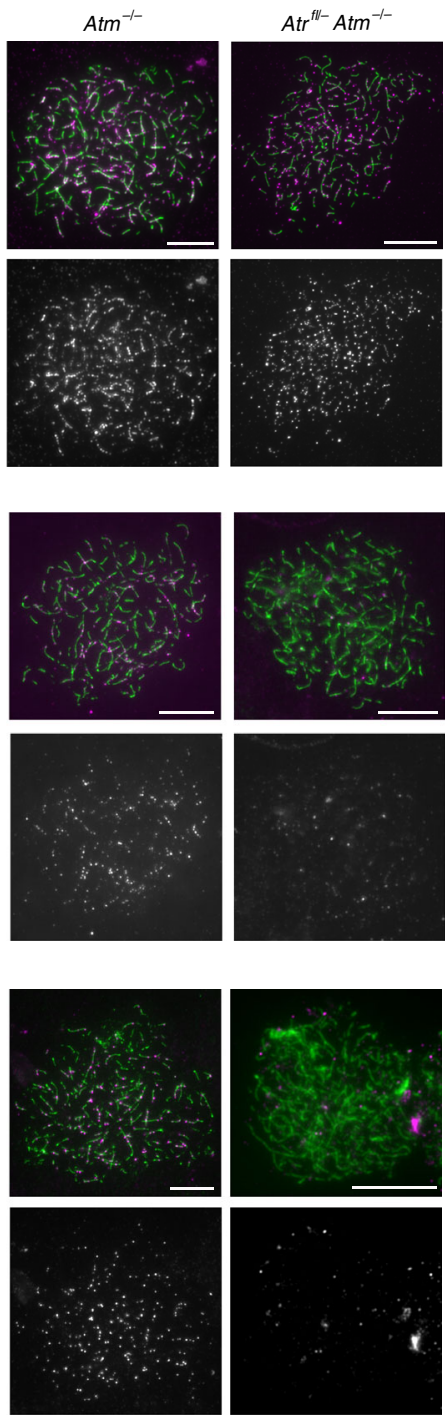

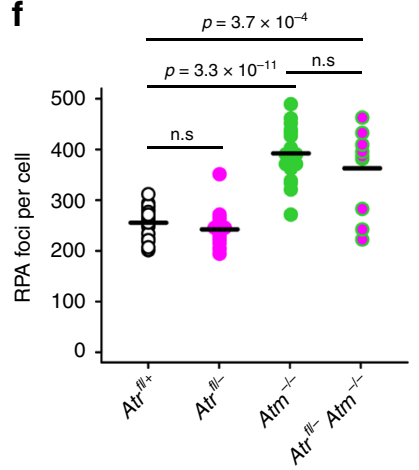

h
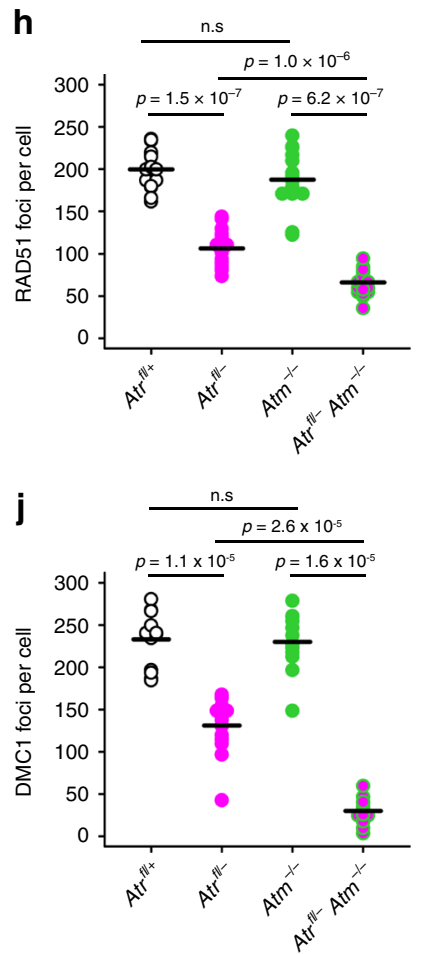
Atr regulates DSB dynamics during later prophase I. We also tested if ATR influences later stages of recombination by quantifying RPA, RAD51 and DMC1 foci throughout prophase I. We restricted our analysis to $A t r r^{f l /}$ males because the extensive chromosome fragmentation after leptonema in $\mathrm{Atr}^{\mathrm{fl} /-} \mathrm{Atm}^{-/-}$ testes prevented a comparison of ATR and ATM functions at these later stages. Interestingly, while RPA counts in Atr fl/- males were equivalent to those in controls at late leptonema (Fig. 4e, f), they were reduced at mid zygonema (Fig. 5a-c). RAD51 and DMC1 counts were also lower in $A t^{f l /-}$ than control males at this stage (Fig. 5c; Supplementary Fig. 3a-d). At early pachynema, DSB markers were assayed both on the autosomes and on the X chromosome, focusing initially on cells with normal autosomal synapsis. Relative to control males, in Atr fl/- males RPA, RAD51 and DMC1 counts were reduced on the autosomes (Fig. 5d-f; Supplementary Fig. 3e-h) and on the X chromosome (Fig. 5g, h, j; Supplementary Fig. 3i-1). The recombination defect in Atr fl/males therefore affects RAD51 and DMC1 at leptonema, and all three DSB markers at mid zygonema and early pachynema.

In mice, synapsis is dependent upon recombination $35,36,49,50$. Asynapsis in Atr ${ }^{f l-}$ males could therefore result not only from SC defects per se (Fig. 3), but also from aberrant recombination. We therefore asked whether early pachytene $A t_{r}^{f l /-}$ spermatocytes with autosomal asynapsis exhibit alterations in DSB marker counts relative to Atr fll- spermatocytes with normal autosomal synapsis. We used RPA as a representative DSB marker, and determined counts on the $\mathrm{X}$ chromosome as an indication of recombination levels. Since it can be obscured by asynapsed autosomes, the $\mathrm{X}$ chromosome was identified using Slx DNA FISH. In Atr fl/- cells with autosomal asynapsis (Fig. 5i) Xchromosome RPA counts were lower than those in Atr $\mathrm{fl}^{\mathrm{l} / \mathrm{-}}$ cells exhibiting normal autosomal synapsis (Fig. 5h, j). Greater alterations in recombination markers therefore correlate with the asynapsis phenotype in Atr fl/- males.

To further define ATR roles later in recombination, we examined the intermediate recombination marker RNF212, a RING-family E3 ligase that localizes to sites of synapsis and is implicated in designation of crossovers ${ }^{51}$. Interestingly, RNF212 focus counts were higher in Atrofl- cells relative to controls (Fig. 6a-d). The elevation was observed in cells with normal synapsis as well as those with autosomal asynapsis. Thus, while deletion of ATR causes a reduction in RPA, RAD51 and DMC1 counts at early pachynema, it leads to an elevation in RNF212 counts at this stage.

\section{Discussion}

The functions of ATR in mammalian meiosis have been unclear. We show here that ATR is required during unperturbed meiosis to regulate chromosome axis integrity, synapsis and recombination. Atr haploinsufficiency compromises the DNA damage response during mitosis ${ }^{13}$. We did not observe haploinsufficiency phenotypes in male meiosis, possibly because they are too mild to detect by our approaches. Alternatively, since ATR expression in the testis far exceeds that in other tissues ${ }^{21}$, Atr haploinsufficiency may be better tolerated during meiosis than mitosis. As is the case in mitosis ${ }^{1}$, in meiosis ATR has roles that are both shared and distinct from ATM. Differences in ATR and ATM functions are likely explained by the contrasting substrate specificities ${ }^{1}$ and meiotic expression profiles ${ }^{24}$ of these kinases.

Deletion of Atr causes mid-pachytene germ cell elimination. This phenotype is also observed in mice deficient in all three DNA damage-regulated PIKKs. Multiple lines of evidence suggest that PIKK depletion in these mice is efficient. Nevertheless, the use of conditional alleles means that residual PIKK activity may be present and sufficient for checkpoint maintenance. Setting aside this caveat, our findings do not exclude a contribution of PIKKs to mid-pachytene elimination in synapsis and recombination mutants. However, they do confirm the existence of additional mechanisms that can trigger elimination. Under such circumstances, mid-pachytene failure is likely caused by defective $\mathrm{MSCI}^{27}$. We suggest that the coexistence of multiple overlapping surveillance mechanisms during prophase I in males explains why checkpoint responses are more robust than those in females ${ }^{52,53}$.

Like ATM ${ }^{54-56}$, ATR regulates homologous synapsis. ATR can promote synapsis independently of meiotic DSB formation, presumably through modification of SC proteins. Among the many SC components, HORMAD1/2 and SMC3 are established ATR phosphortargets ${ }^{16,57,58}$. While additional SC candidates no doubt exist, HORMAD1 is of particular interest, because this protein can also promote synapsis in the absence of recombination $^{34}$. We find that in wild-type males, asynapsis of the XY pair occurs at similar frequency to that of all autosomal pairs combined. This bias towards XY asynapsis may be attributable to the small length and terminal location of the PAR. Notably however, in Atr mutants, the bias is exaggerated, such that asynapsis of the XY bivalent occurs at higher frequency than that of all autosomes combined. The PAR is unusual, undergoing late DSB formation and synapsis ${ }^{33}$, and being enriched for repressive chromatin marks not observed at the termini of autosomal bivalents ${ }^{59,60}$. We suggest that ATR promotes one or more of these unique properties to ensure successful XY interactions.

DSB frequency increases drastically when ATM is missing ${ }^{40}$, but ATR deficiency has little if any effect on SPO11-oligo complex amounts in either ATM-proficient or ATM-deficient backgrounds. Thus, we conclude that ATR does not contribute significantly to control of DSB numbers. Atm mutants also exhibit an increase in the lengths of SPO11 oligos ${ }^{40}$.

Fig. 4 ATR ablation does not alter DSB levels but leads to reduction in leptotene recombinase counts. a-d Analysis of SPO11-oligo complexes in P13 testes. SPO11 was immunoprecipitated from whole-testis extracts and SPO11-associated oligos were end-labeled with terminal deoxynucleotidyl transferase and $\left[\alpha^{-32} \mathrm{P}\right] \mathrm{dCTP}$, then either separated on SDS-PAGE gels followed by autoradiography (a) and western blotting with anti-SPO11 antibody (b) or digested with proteinase $\mathrm{K}$ and resolved on denaturing polyacrylamide sequencing gels (c; background-subtracted lane traces in $\mathbf{d}$ ). A representative experiment is shown. An additional Atr $\mathrm{fl}^{\mathrm{I}+} \mathrm{Cre}$ - control is shown to demonstrate that the Ngn3-Cre transgene does not influence SPO11-oligo levels. In $\mathbf{a}$, b, the bar indicates SPO11-oligo complexes, arrowhead indicates the immunoglobulin heavy chain and asterisk marks non-specific labeling; ND not determined. Each panel shows lanes from the same exposure of a single western blot or autoradiograph, with intervening lanes omitted. For quantitation, SPO11-oligo

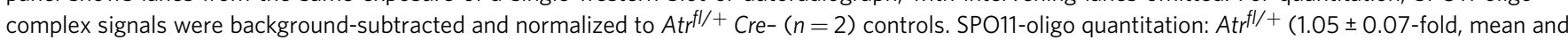
s.d., $n=2$ males), Atr ${ }^{f l /-}(1.17 \pm 0.22$-fold, $n=4$ males $)$, Atm $^{-/-}(14.58 \pm 5.24$-fold, $n=3$ males $)$ and Atr ${ }^{f l /}-$ Atm $^{-/}$- males (11.38 \pm 13.15 -fold $)$. The reduced SPO11 protein levels in $\mathrm{Atm}^{-/-}$were previously documented ${ }^{40,79}$, but the molecular basis is not understood. e-j Analysis of leptotene focus counts using SYCP3 (green) and early recombination markers (magenta): RPA (e, f), RAD51 ( $\mathbf{g}, \mathbf{h})$ and DMC1 (i, j) in Atr fl/ + males $(n=2$ males; 27 cells for RPA, 19 cells for RAD51, 14 cells for DMC1), Atr ${ }^{f l /-}$ males ( $n=2$ males; 21 cells for RPA, 19 cells for RAD51, 13 cells for DMC1), Atm ${ }^{-/-}$males $(n=2$ males; 22 cells for RPA, 15 cells for RAD51, 13 cells for DMC1) and Atr fl/- Atm ${ }^{-/-}$males ( $n=2$ males; 10 cells for RPA, 20 cells for RAD51, 13 cells for DMC1). RPA counts are not significantly different between $A t_{r} f l-$ males (mean 249 foci) and Atr ${ }^{f l} /+$ males without the Ngn3-Cre transgene (mean 227 foci, $n=2$ males, 17 cells), and are thus not influenced by Atr haploinsufficiency. Mean and $p$ values (Mann-Whitney test) indicated. Scale bars $10 \mu \mathrm{m}$ 
a

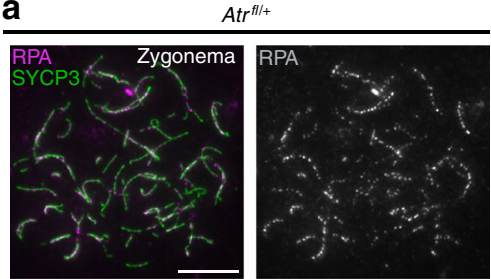

d
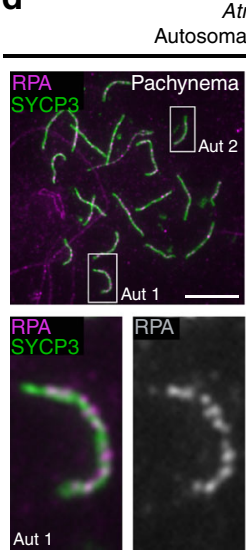

g
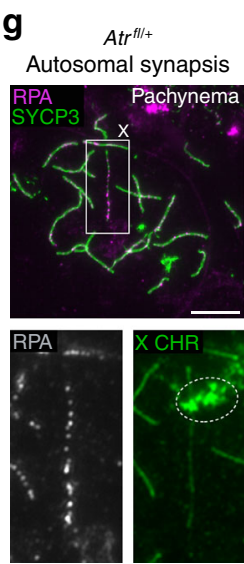

b

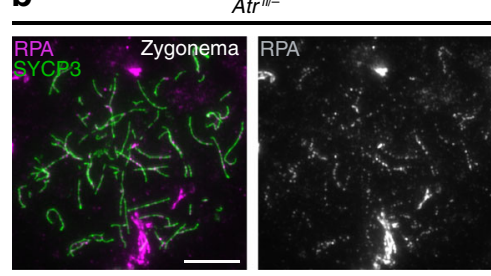

e

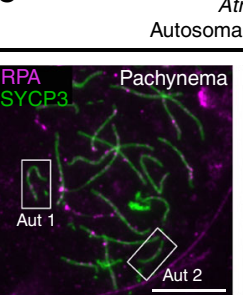

$A t r^{f l l}$
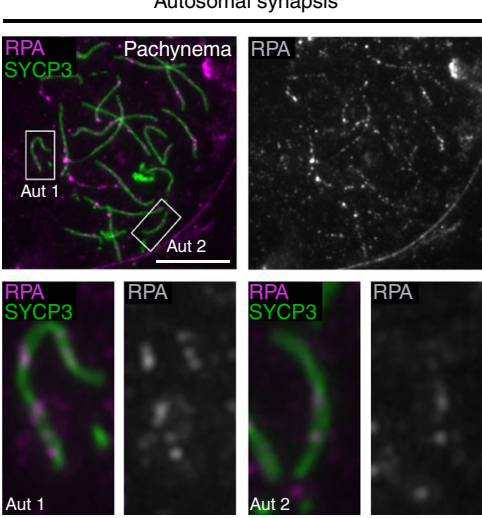

i $\quad \operatorname{tr} t^{f / L}$

Autosomal asynapsis
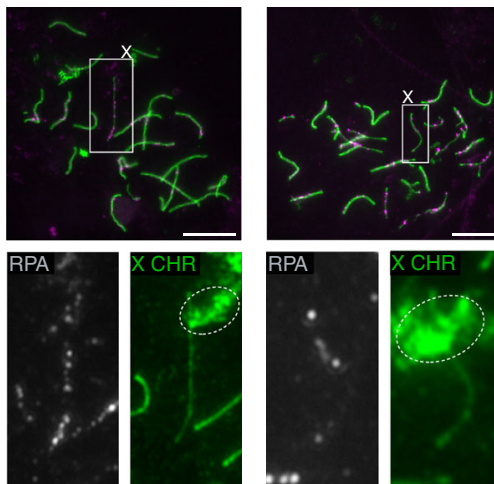

C
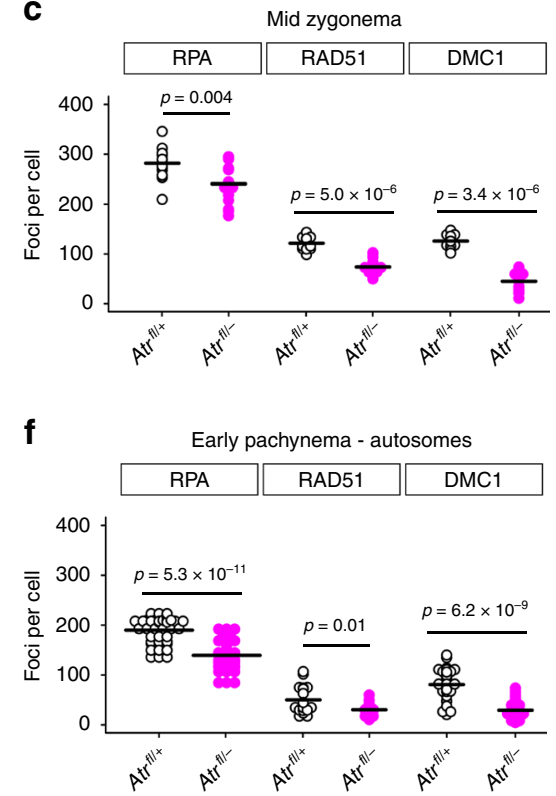

Fig. 5 ATR regulates DSB marker counts during zygonema and pachynema. a, b RPA (magenta) and SYCP3 (green) immunostaining in Atr ${ }^{f l /+}$ and $A t r f l /-$ males. c RPA, RAD51 and DMC1 counts at mid zygonema in $A$ tr $^{f l /+}$ males ( $n=2$ males; 16 cells for RPA, 15 cells for RAD51, 15 cells for DMC1) and Atr ${ }^{f l-}$ males ( $n=2$ males; 16 cells for RPA, 15 cells for RAD51, 15 cells for DMC1). d, e Examples of early pachytene cells from Atrfl/t+ and Atrl//- males with normal autosomal synapsis. Two representative autosomes (aut 1 and 2 ) are boxed in upper panels and magnified in lower panels. $\mathbf{f}$ autosomal RPA, RAD51 and DMC1 counts at early pachynema in $\operatorname{Atr}^{\mathrm{fl} /+}$ males ( $n=2$ males; 49 cells for RPA, 19 cells for RAD51, 33 cells for DMC 1$)$ and Atr ${ }^{f l-}$ males $(n=2$ males; 43 cells for RPA, 19 cells for RAD51, 35 cells for DMC1). g, h Early pachytene Atr ${ }^{f l /+}$ and Atrfl/- cells with normal autosomal synapsis and the X chromosome (boxed in upper panels) identified by SIx DNA FISH (dashed circles in lower panels). i Early pachytene Atr ${ }^{f l /}$ - cell with autosomal asynapsis. $\mathbf{j}$ X-chromosome RPA, RAD51 and DMC1 counts at early pachynema in Atr ${ }^{f l+}+$ males ( $n=2$ males; 64 cells for RPA, 49 cells for RAD51, 59 cells for DMC1) and Atr ${ }^{f l /-}$ males ( $n=2$ males; 62 cells for RPA in autosomal synapsis category, 35 cells for RPA in autosomal asynapsis category, 36 cells for RAD51, 53 cells for DMC1). Mean and $p$ values (Mann-Whitney test) indicated. Scale bars $10 \mu \mathrm{m}$

This phenotype is not observed in Atr single mutants, but SPO11oligos become even longer in Atr Atm double mutants. These findings suggest that ATR can partially substitute for ATM in promoting normal nucleolytic processing of DSBs. For example, ATR may influence the $3^{\prime} \rightarrow 5^{\prime}$ exonuclease activity of MRE11, which is known to govern Spo11-oligo length in yeast ${ }^{61}$. CtIP and its yeast ortholog Sae2, MRE11 partners important for DSB processing, are PIKK phosphortargets ${ }^{62-64}$.

Although ATR deficiency does not appear to modulate DSB numbers, Atr mutants do display a substantial reduction in RAD51 and DMC1 focus counts. This finding suggests that ATR specifically promotes the assembly of RAD51 and DMC1 on resected DSBs. It has been suggested that ATR phosphorylates RAD51 ${ }^{65}$. ATR also phosphorylates CHK1 during meiosis ${ }^{66}$, and CHK1 in turn promotes RAD51 loading at DSBs ${ }^{67}$. A nonexclusive alternative is that focus numbers are reduced in the mutant because ATR slows their turnover, for example by inhibiting use of the sister chromatid as a recombination partner ${ }^{5,68}$. Atm single mutants do not display a reduction in RAD51 and $\mathrm{DMC} 1$ foci, but the interpretation of this result is complicated by the fact that the mutants make more DSBs that are thought to often occur in clusters that may not be cytologically distinguishable from single DSBs ${ }^{40,42}$. Thus, whether and how ATM might influence RAD51 and DMC1 assembly has been unclear. 
a

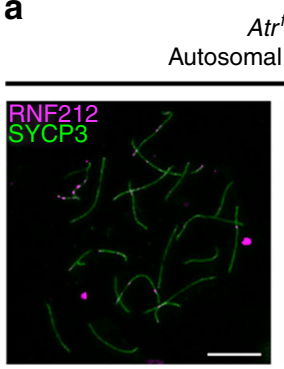

$\operatorname{Atr}^{\mathrm{fl} /+}$

Autosomal synapsis

C

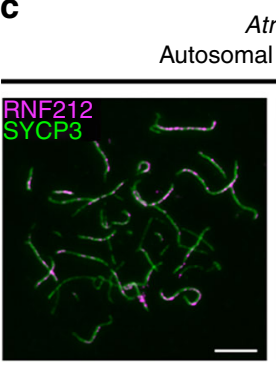

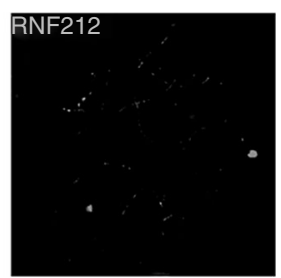

$\operatorname{Atr}^{f l l-}$

asynapsis

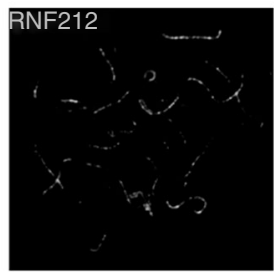

b
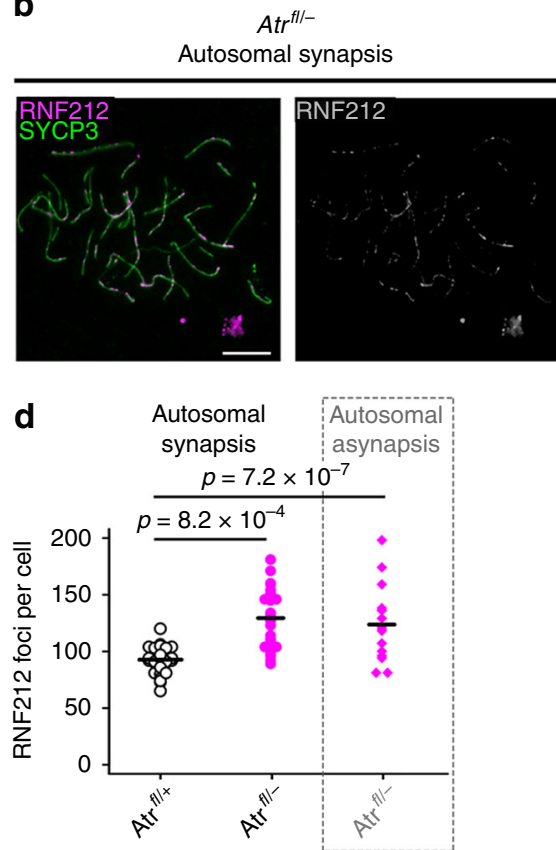

Fig. 6 ATR regulates RNF212 counts during pachynema. a-c RNF212 (magenta) and SYCP3 (green) immunostaining at early pachynema in Atr ${ }^{f l /+}$ and Atr ${ }^{f l}$ - males. d RNF212 counts in Atr fl/+ male ( $n=1$ male; 29 cells) and Atr ${ }^{f l /-}$ male with normal autosomal synapsis $(n=1$ male; 15 cells) or autosomal asynapsis ( $n=1$ males; 25 cells). Mean and $p$ values (Mann-Whitney test) indicated. Scale bars $10 \mu \mathrm{m}$

We find that RAD51 and DMC1 focus counts are lower in the Atr Atm double mutants than in the Atr single mutant without an apparent change in DSB number. This finding suggests that ATM can indeed facilitate assembly of RAD51- and DMC1-containing recombination intermediates, at least in the absence of ATR. If so, ATM may promote RAD51 and DMC1 assembly directly, or may do so indirectly by fostering DSB resection, as its ortholog Tel1 does in yeast ${ }^{2}, 46$. ATM may also influence the lifespan of RAD51 and DMC1 foci via effects on sister chromatid recombination.

Later in prophase I, spermatocytes lacking ATR exhibit lower counts not only for RAD51 and DMC1 but also for RPA. In contrast, focus counts for the intermediate recombination marker RNF212 are elevated. These changes could imply additional functions for ATR in later processing of recombination intermediates and/or crossover designation. Alternatively, the reduction in RPA, RAD51 and DMC1 may indicate the presence of fewer unrepaired DSBs. This possibility is not precluded by the RNF212 findings, because RNF212 can localize to the SC in the absence of recombination ${ }^{51}$. Reduced DSB number in Atr mutants could result from premature repair using the sister chromatid ${ }^{5}$, or from a failure to induce new DSBs on asynapsed chromosomes ${ }^{69}$. Interestingly, the ATR substrates HORMAD1/2 are implicated both in inhibiting sister chromatid recombination 70,71 and in promoting ongoing DSB formation $^{70,72}$. Our data are less consistent with a defect in generating new DSBs on asynapsed chromosomes, because SPO11-oligo data showed no reduction in DSB formation in Atr mutants. Furthermore, in these mutants decreased RPA counts were also observed on synapsed autosomes, and the magnitude of the decrease was similar to that seen for the asynapsed $\mathrm{X}$ chromosome. If there are fewer DSBs during pachynema in the Atr mutant, then the increase in RNF212 foci seen at this stage could imply a role for ATR in regulating RNF212 abundance at the SC independently of recombination.

Whether ATR regulates crossover formation and designation is currently unclear. Analyses of male mice with a hypomorphic Atr mutation or with pharmacologically inhibited ATR suggest that this kinase is required for crossovers ${ }^{73}$. We could not analyze crossover formation in our Atr mutants because crossover markers appear after the point of germ cell elimination in these mice (Supplementary Fig. 4). Conditional deletion of Atr in the female mouse germline, where recombination defects incur germ cell elimination later in meiosis ${ }^{74}$, may help to resolve this point.

\section{Methods}

Animal experiments. All mice were maintained under UK Home Office Regulations, UK Animals (Scientific Procedures) Act 1986, and according to ethical guidelines at the National Institute for Medical Research (NIMR) and the Francis Crick Institute Mill Hill laboratory. Permission for animal experiments was granted by The Crick Biological Research Facility Strategic Oversight Committee (BRF-SOC) incorporating Animal Welfare and Ethical Review Body (AWERB) (Project Licence P8ECF28D9 granted by the Secretary of State). Genetically modified models are previously published and are maintained on a predominantly C57BL/6 background: Ngn3-Cre ${ }^{20}$, Stra8-Cre ${ }^{18}$, Atr $^{14}$, Atr flox ${ }^{75}$, Atm $^{76}$, Atm flox ${ }^{31}, S p o 11^{36}$ and $D m c 1^{49}$. Prkdc scid/scid mice were obtained from the Jackson Labs and are maintained on an NOD background. Littermate controls were used where possible.

Immunofluorescence, focus counting and western blotting. Immunofluorescence experiments on surface spread spermatocytes were carried out as previously described ${ }^{28}$. In brief, cells were permeabilized for $10 \mathrm{~min}$ in $0.05 \%$ Triton X-100 and fixed for $1 \mathrm{~h}$ minimum in $2 \%$ formaldehyde, $0.02 \%$ SDS in phosphate-buffered saline (PBS). Slides were rinsed in distilled water, air dried and blocked in PBT (0.15\% bovine serum albumin, 0.10\% TWEEN-20 in PBS) for $1 \mathrm{~h}$ Slides were incubated with the following antibodies in a humid chamber overnight at $37^{\circ} \mathrm{C}$ : guinea-pig anti-SYCP3 (made in-house) 1:500, rabbit anti-SYCP3 (Abcam ab-15092) 1:100, rabbit anti-ATR (Cell Signalling \#2790) 1:50, mouse anti- $\gamma \mathrm{H} 2 \mathrm{AX}$ (Millipore 05-636) 1:100, rabbit anti-HORMAD2 (Tóth lab) 1:100, guinea-pig anti-SYCE2 (gift from Howard Cooke/Ian Adams) 1:800, human anti-centromere (CREST ab gift from Bill Earnshaw) 1:1000, rabbit anti-RPA (Abcam ab-2175) 1:100 anti-rabbit RAD51 (Calbiochem PC130) 1:25, goat anti-DMC1 (Santa Cruz sc-8973) 1:25. Western blotting was carried out as previously described ${ }^{77}$ (Supplementary Fig. 6). Counting of recombination protein foci was performed manually, considering only foci that colocalized with axial elements.

Meiotic staging. The presence of asynapsis can make discrimination between zygotene cells and pachytene cells with asynapsis challenging. We used the following criteria (Supplementary Fig. 5). (1) DAPI (4',6-diamidino-2-phenylindole) staining: at zygonema DAPI staining is bright and centromeres are clustered in a 
few subdomains, while in pachynema DAPI staining is heterogeneous, with euchromatin stained faintly and centromeres stained brightly and forming multiple subdomains. (2) The length and thickness of axial elements/SCs: axial elements are long and thin in zygonema, and are not yet fully formed, but they are shorter and thicker and fully formed in pachynema. (3) Chromosomal asynchrony: in contrast to zygotene cells, pachytene cells with asynapsis exhibit asynchrony in synapsis between individual bivalents, i.e., the coexistence of completely asynapsed bivalents and multiple fully synapsed bivalents. For comparison of synapsis between Spo11 -/- males and Atr ${ }^{f l /}$-Spo11 $1^{-/-}$males, pachytene cells were identified by virtue of having fully developed axial elements.

RNA, DNA FISH and SPO11-oligo analysis. FISH was carried out with digoxigenin-labeled probes as previously described ${ }^{78}$. CHORI BAC probe, RP24204018 (CHORI) was used for Scml2 RNA FISH, RP23-470D15 for Slx DNA FISH, and RP24-502P5 for Sly DNA FISH. Analyses of abundance of SPO11-oligo complexes and sizes of SPO11 oligos were performed as previously described ${ }^{40,44}$.

Microscopy. Imaging was performed using an Olympus IX70 inverted microscope with a 100-W mercury arc lamp. For chromosome spread and RNA FISH imaging, an Olympus UPlanApo $100 \times / 1.35 \mathrm{NA}$ oil immersion objective was used. For testis section imaging, an Olympus UPlanApo $40 \times / 0.75$ NA objective was used. A Deltavision RT computer-assisted Photometrics CoolsnapHQ CCD camera with an ICX285 Progressive scan CCD image sensor was utilized for image capture. Then, 8 or 16 -bit $(512 \times 512$ or $1024 \times 1024$ pixels $)$ raw images of each channel were captured and later processed using Fiji software.

Statistics. Statistical calculations were performed using GraphPad Prism 6.0. For comparison of two genotypes, Mann-Whitney test or $t$-tests were performed.

Data availability. The data that support the findings of this study are available from the corresponding author upon request.

Received: 18 May 2017 Accepted: 24 May 2018

Published online: 05 July 2018

\section{References}

1. Marechal, A. \& Zou, L. DNA damage sensing by the ATM and ATR kinases. Cold Spring Harb. Perspect. 5, a012716 (2013).

2. Joshi, N., Brown, M. S., Bishop, D. K. \& Borner, G. V. Gradual implementation of the meiotic recombination program via checkpoint pathways controlled by global DSB levels. Mol. Cell 57, 797-811 (2015).

3. Kurzbauer, M. T., Uanschou, C., Chen, D. \& Schlogelhofer, P. The recombinases DMC1 and RAD51 are functionally and spatially separated during meiosis in Arabidopsis. Plant Cell 24, 2058-2070 (2012).

4. Grushcow, J. M. et al. Saccharomyces cerevisiae checkpoint genes MEC1, RAD17 and RAD24 are required for normal meiotic recombination partner choice. Genetics 153, 607-620 (1999).

5. Carballo, J. A., Johnson, A. L., Sedgwick, S. G. \& Cha, R. S. Phosphorylation of the axial element protein Hop1 by Mec1/Tell ensures meiotic interhomolog recombination. Cell 132, 758-770 (2008).

6. Carpenter, A. T. Recombination nodules and synaptonemal complex in recombination-defective females of Drosophila melanogaster. Chromosoma 75, 259-292 (1979).

7. Falk, J. E., Chan, A. C., Hoffmann, E. \& Hochwagen, A. A Mec1- and PP4dependent checkpoint couples centromere pairing to meiotic recombination. Dev. Cell 19, 599-611 (2010).

8. Abdu, U., Brodsky, M. \& Schupbach, T. Activation of a meiotic checkpoint during Drosophila oogenesis regulates the translation of Gurken through Chk2/Mnk. Curr. Biol. 12, 1645-1651 (2002).

9. Lydall, D., Nikolsky, Y., Bishop, D. K. \& Weinert, T. A meiotic recombination checkpoint controlled by mitotic checkpoint genes. Nature 383, 840-843 (1996).

10. Murakami, H. \& Nurse, P. Meiotic DNA replication checkpoint control in fission yeast. Genes Dev. 13, 2581-2593 (1999).

11. Blitzblau, H. G. \& Hochwagen, A. ATR/Mecl prevents lethal meiotic recombination initiation on partially replicated chromosomes in budding yeast. eLife 2, e00844 (2013).

12. O’Driscoll, M., Ruiz-Perez, V. L., Woods, C. G., Jeggo, P. A. \& Goodship, J. A. A splicing mutation affecting expression of ataxia-telangiectasia and Rad3related protein (ATR) results in Seckel syndrome. Nat. Genet. 33, 497-501 (2003).

13. O’Driscoll, M., Dobyns, W. B., van Hagen, J. M. \& Jeggo, P. A. Cellular and clinical impact of haploinsufficiency for genes involved in ATR signaling. Am. J. Human Genet. 81, 77-86 (2007).

14. Brown, E. J. \& Baltimore, D. ATR disruption leads to chromosomal fragmentation and early embryonic lethality. Genes Dev. 14, 397-402 (2000).
15. de Klein, A. et al. Targeted disruption of the cell-cycle checkpoint gene ATR leads to early embryonic lethality in mice. Curr. Biol. 10, 479-482 (2000).

16. Royo, $\mathrm{H}$. et al. ATR acts stage specifically to regulate multiple aspects of mammalian meiotic silencing. Genes Dev. 27, 1484-1494 (2013).

17. Brown, E. J. \& Baltimore, D. Essential and dispensable roles of ATR in cell cycle arrest and genome maintenance. Genes Dev. 17, 615-628 (2003).

18. Sadate-Ngatchou, P. I., Payne, C. J., Dearth, A. T. \& Braun, R. E. Cre recombinase activity specific to postnatal, premeiotic male germ cells in transgenic mice. Genesis 46, 738-742 (2008).

19. Zheng, K. \& Wang, P. J. Blockade of pachytene piRNA biogenesis reveals a novel requirement for maintaining post-meiotic germline genome integrity. PLoS Genet. 8, e1003038 (2012).

20. Schonhoff, S. E., Giel-Moloney, M. \& Leiter, A. B. Neurogenin 3-expressing progenitor cells in the gastrointestinal tract differentiate into both endocrine and non-endocrine cell types. Dev. Biol. 270, 443-454 (2004).

21. Keegan, K. S. et al. The Atr and Atm protein kinases associate with different sites along meiotically pairing chromosomes. Genes Dev. 10, 2423-2437 (1996).

22. Lammers, J. H. et al. The gene encoding a major component of the lateral elements of synaptonemal complexes of the rat is related to X-linked lymphocyte-regulated genes. Mol. Cell. Biol. 14, 1137-1146 (1994).

23. Barchi, M. et al. Surveillance of different recombination defects in mouse spermatocytes yields distinct responses despite elimination at an identical developmental stage. Mol. Cell. Biol. 25, 7203-7215 (2005).

24. Bellani, M. A., Romanienko, P. J., Cairatti, D. A. \& Camerini-Otero, R. D. SPO11 is required for sex-body formation, and Spo11 heterozygosity rescues the prophase arrest of Atm-/- spermatocytes. J. Cell Sci. 118, 3233-3245 (2005).

25. Mahadevaiah, S. K. et al. Extensive meiotic asynapsis in mice antagonises meiotic silencing of unsynapsed chromatin and consequently disrupts meiotic sex chromosome inactivation. J. Cell Biol. 182, 263-276 (2008).

26. Pacheco, S. et al. The ATM signaling cascade promotes recombinationdependent pachytene arrest in mouse spermatocytes. PLoS Genet. 11, e1005017 (2015).

27. Royo, H. et al. Evidence that meiotic sex chromosome inactivation is essential for male fertility. Curr. Biol. 20, 2117-2123 (2010).

28. Turner, J. M. et al. Silencing of unsynapsed meiotic chromosomes in the mouse. Nat. Genet. 37, 41-47 (2005).

29. Fernandez-Capetillo, O., Liebe, B., Scherthan, H. \& Nussenzweig, A. H2AX regulates meiotic telomere clustering. J. Cell. Biol. 163, 15-20 (2003)

30. Araki, R. et al. Nonsense mutation at Tyr-4046 in the DNA-dependent protein kinase catalytic subunit of severe combined immune deficiency mice. Proc. Natl Acad. Sci. USA 94, 2438-2443 (1997).

31. Zha, S., Sekiguchi, J., Brush, J. W., Bassing, C. H. \& Alt, F. W. Complementary functions of ATM and H2AX in development and suppression of genomic instability. Proc. Natl Acad. Sci. USA 105, 9302-9306 (2008).

32. Gurley, K. E. \& Kemp, C. J. Synthetic lethality between mutation in Atm and DNA-PK(cs) during murine embryogenesis. Curr. Biol. 11, 191-194 (2001).

33. Kauppi, L. et al. Distinct properties of the XY pseudoautosomal region crucial for male meiosis. Science 331, 916-920 (2011).

34. Daniel, K. et al. Meiotic homologue alignment and its quality surveillance are controlled by mouse HORMAD1. Nat. Cell Biol. 13, 599-610 (2011).

35. Romanienko, P. J. \& Camerini-Otero, R. D. The mouse Spol1 gene is required for meiotic chromosome synapsis. Mol. Cell 6, 975-987 (2000).

36. Baudat, F., Manova, K., Yuen, J. P., Jasin, M. \& Keeney, S. Chromosome synapsis defects and sexually dimorphic meiotic progression in mice lacking Spo11. Mol. Cell 6, 989-998 (2000).

37. Costa, Y. et al. Two novel proteins recruited by synaptonemal complex protein 1 (SYCP1) are at the centre of meiosis. J. Cell Sci. 118, 2755-2762 (2005)

38. Joyce, E. F. et al. Drosophila ATM and ATR have distinct activities in the regulation of meiotic DNA damage and repair. J. Cell Biol. 195, 359-367 (2011).

39. Zhang, L., Kim, K. P., Kleckner, N. E. \& Storlazzi, A. Meiotic double-strand breaks occur once per pair of (sister) chromatids and, via Mec1/ATR and Tel1/ATM, once per quartet of chromatids. Proc. Natl Acad. Sci. USA 108, 20036-20041 (2011)

40. Lange, J. et al. ATM controls meiotic double-strand-break formation. Nature 479, 237-240 (2011).

41. Carballo, J. A. et al. Budding yeast ATM/ATR control meiotic double-strand break (DSB) levels by down-regulating Rec114, an essential component of the DSB-machinery. PLoS Genet. 9, e1003545 (2013).

42. Garcia, V., Gray, S., Allison, R. M., Cooper, T. J. \& Neale, M. J. Tel1(ATM)mediated interference suppresses clustered meiotic double-strand-break formation. Nature 520, 114-118 (2015)

43. Gray, S., Allison, R. M., Garcia, V., Goldman, A. S. \& Neale, M. J. Positive regulation of meiotic DNA double-strand break formation by activation of the DNA damage checkpoint kinase Mec1(ATR). Open Biol. 3, 130019 (2013).

44. Neale, M. J., Pan, J. \& Keeney, S. Endonucleolytic processing of covalent protein-linked DNA double-strand breaks. Nature 436, 1053-1057 (2005). 
45. Terasawa, M., Ogawa, T., Tsukamoto, Y. \& Ogawa, H. Sae2p phosphorylation is crucial for cooperation with Mre11p for resection of DNA double-strand break ends during meiotic recombination in Saccharomyces cerevisiae. Genes Genet. Syst. 83, 209-217 (2008).

46. Mimitou, E. P., Yamada, S. \& Keeney, S. A global view of meiotic doublestrand break end resection. Science 355, 40-45 (2017).

47. Mohibullah, N. \& Keeney, S. Numerical and spatial patterning of yeast meiotic DNA breaks by Tel1. Genome Res. 27, 278-288 (2017).

48. Zou, L. \& Elledge, S. J. Sensing DNA damage through ATRIP recognition of RPA-ssDNA complexes. Science 300, 1542-1548 (2003).

49. Pittman, D. L. et al. Meiotic prophase arrest with failure of chromosome synapsis in mice deficient for Dmc1, a germline-specific RecA homolog. Mol. Cell 1, 697-705 (1998).

50. Yoshida, K. et al. The mouse RecA-like gene Dmcl is required for homologous chromosome synapsis during meiosis. Mol. Cell 1, 707-718 (1998).

51. Reynolds, A. et al. RNF212 is a dosage-sensitive regulator of crossing-over during mammalian meiosis. Nat. Genet. 45, 269-278 (2013).

52. Morelli, M. A. \& Cohen, P. E. Not all germ cells are created equal: aspects of sexual dimorphism in mammalian meiosis. Reproduction 130, 761-781 (2005).

53. Hunt, P. A. \& Hassold, T. J. Sex matters in meiosis. Science 296, 2181-2183 (2002).

54. Barchi, M. et al. ATM promotes the obligate XY crossover and both crossover control and chromosome axis integrity on autosomes. PLoS Genet. 4, e1000076 (2008).

55. Xu, Y. et al. Targeted disruption of ATM leads to growth retardation, chromosomal fragmentation during meiosis, immune defects, and thymic lymphoma. Genes Dev. 10, 2411-2422 (1996).

56. Barlow, C. et al. Atm deficiency results in severe meiotic disruption as early as leptonema of prophase I. Development 125, 4007-4017 (1998).

57. Luo, H. et al. Regulation of intra-S phase checkpoint by ionizing radiation (IR)-dependent and IR-independent phosphorylation of SMC3. J. Biol. Chem. 283, 19176-19183 (2008).

58. Fukuda, T. et al. Phosphorylation of chromosome core components may serve as axis marks for the status of chromosomal events during mammalian meiosis. PLoS Genet. 8, el002485 (2012).

59. Turner, J. M., Burgoyne, P. S. \& Singh, P. B. M31 and macroH2A1.2 colocalise at the pseudoautosomal region during mouse meiosis. J. Cell Sci. 114, 3367-3375 (2001).

60. Peters, A. H. et al. Loss of the Suv39h histone methyltransferases impairs mammalian heterochromatin and genome stability. Cell 107, 323-337 (2001).

61. Garcia, V., Phelps, S. E., Gray, S. \& Neale, M. J. Bidirectional resection of DNA double-strand breaks by Mrel1 and Exol. Nature 479, 241-244 (2011).

62. Peterson, S. E. et al. Activation of DSB processing requires phosphorylation of CtIP by ATR. Mol. Cell 49, 657-667 (2013).

63. Cartagena-Lirola, H., Guerini, I., Viscardi, V., Lucchini, G. \& Longhese, M. P. Budding yeast Sae2 is an in vivo target of the Mec1 and Tell checkpoint kinases during meiosis. Cell Cycle 5, 1549-1559 (2006).

64. Baroni, E., Viscardi, V., Cartagena-Lirola, H., Lucchini, G. \& Longhese, M. P. The functions of budding yeast Sae2 in the DNA damage response require Mec1- and Tel1-dependent phosphorylation. Mol. Cell. Biol. 24, 4151-4165 (2004).

65. Flott, S. et al. Regulation of Rad51 function by phosphorylation. EMBO Rep. 12, 833-839 (2011).

66. Fedoriw, A. M., Menon, D., Kim, Y., Mu, W. \& Magnuson, T. Key mediators of somatic ATR signaling localize to unpaired chromosomes in spermatocytes. Development 142, 2972-2980 (2015).

67. Sorensen, C. S. et al. The cell-cycle checkpoint kinase Chk1 is required for mammalian homologous recombination repair. Nat. Cell Biol. 7, 195-201 (2005).

68. Goldfarb, T. \& Lichten, M. Frequent and efficient use of the sister chromatid for DNA double-strand break repair during budding yeast meiosis. PLoS Biol. 8, e1000520 (2010)

69. Kauppi, L. et al. Numerical constraints and feedback control of double-strand breaks in mouse meiosis. Genes Dev. 27, 873-886 (2013).

70. Wojtasz, L. et al. Mouse HORMAD1 and HORMAD2, two conserved meiotic chromosomal proteins, are depleted from synapsed chromosome axes with the help of TRIP13 AAA-ATPase. PLoS Genet. 5, e1000702 (2009).

71. Shin, Y. H., McGuire, M. M. \& Rajkovic, A. Mouse HORMAD1 is a meiosis $\mathrm{i}$ checkpoint protein that modulates DNA double- strand break repair during female meiosis. Biol. Reprod. 89, 29 (2013).

72. Keeney, S., Lange, J. \& Mohibullah, N. Self-organization of meiotic recombination initiation: general principles and molecular pathways. Annu. Rev. Genet. 48, 187-214 (2014).

73. Pacheco, S. et al. ATR is required to complete meiotic recombination in mice. Nat. Commun. https://doi.org/10.1038/s41467-018-04851-z (2018).
74. Di Giacomo, M. et al. Distinct DNA-damage-dependent and -independent responses drive the loss of oocytes in recombination-defective mouse mutants. Proc. Natl Acad. Sci. USA 102, 737-742 (2005).

75. Ruzankina, Y. et al. Deletion of the developmentally essential gene ATR in adult mice leads to age-related phenotypes and stem cell loss. Cell Stem Cell $\mathbf{1}$, 113-126 (2007).

76. Barlow, C. et al. Atm-deficient mice: a paradigm of ataxia telangiectasia. Cell 86, 159-171 (1996).

77. Hamer, G., Kal, H. B., Westphal, C. H., Ashley, T. \& de Rooij, D. G. Ataxia telangiectasia mutated expression and activation in the testis. Biol. Reprod. 70, 1206-1212 (2004)

78. Mahadevaiah, S. K., Costa, Y. \& Turner, J. M. Using RNA FISH to study gene expression during mammalian meiosis. Methods Mol. Biol. 558, 433-444 (2009).

79. Bellani, M. A., Boateng, K. A., McLeod, D. \& Camerini-Otero, R. D. The expression profile of the major mouse SPO11 isoforms indicates that SPO11beta introduces double strand breaks and suggests that SPO11alpha has an additional role in prophase in both spermatocytes and oocytes. Mol. Cell. Biol. 30, 4391-4403 (2010).

\section{Acknowledgements}

We are grateful to Bill Earnshaw for providing the CREST anti-centromere antibody, Howard Cooke, Ian Adams for providing the SYCE2 antibody, Paula Cohen for providing the anti-MLH3 antibody, Neil Hunter for the anti-RNF212 antibody, Eric Brown for providing the Atr flox and null mice, members of the Turner laboratory for critical reading of the manuscript and the Francis Crick Institute Technology platforms for excellent assistance. This work was supported by the Francis Crick Institute which receives its core funding from Cancer Research UK (FC001193), the UK Medical Research Council (FC001193) and the Wellcome Trust (FC001193). Work in the Keeney lab is supported in part by the US National Institutes of Health grant R35 GM118092; J.L. was supported in part by American Cancer Society fellowship PF-12-157-01-DMC. S.P., A.M.-L. and I.R. are supported by the Ministerio de Ciencia e Innovación (BFU201680370-P)

\section{Author contributions}

A.W., O.O., V.M. and J.M.A.T. performed animal generation and genotyping; A.W., S.K. M., M.S., S.P., A.M.-L. and J.M.A.T. performed immunofluorescence; A.W., E.E. and V. M. performed western blots; A.W. and S.K.M. performed RNA FISH; T.H. performed DNA FISH; J.L. and S.K. performed SPO11-oligo experiments; A.W. and D.d.R. performed histology; A.W. and J.Z. performed data plotting and statistics; A.T. supplied HORMAD2 antibody. J.M.A.T. wrote the manuscript with critical input from A.T., S.K., I.R. and Turner lab members.

\section{Additional information}

Supplementary Information accompanies this paper at https://doi.org/10.1038/s41467018-04850-0.

Competing interests: The authors declare no competing interests.

Reprints and permission information is available online at http://npg.nature.com/ reprintsandpermissions/

Publisher's note: Springer Nature remains neutral with regard to jurisdictional claims in published maps and institutional affiliations.

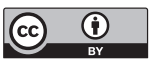

Open Access This article is licensed under a Creative Commons Attribution 4.0 International License, which permits use, sharing, adaptation, distribution and reproduction in any medium or format, as long as you give appropriate credit to the original author(s) and the source, provide a link to the Creative Commons license, and indicate if changes were made. The images or other third party material in this article are included in the article's Creative Commons license, unless indicated otherwise in a credit line to the material. If material is not included in the article's Creative Commons license and your intended use is not permitted by statutory regulation or exceeds the permitted use, you will need to obtain permission directly from the copyright holder. To view a copy of this license, visit http://creativecommons.org/ licenses/by/4.0/.

(C) The Author(s) 2018 